04

\title{
Генерация суммарной частоты от тонкого цилиндрического слоя
}

\author{
(C) A.A. Шамына, B.Н. Капшай \\ Гомельский государственный университет им. Ф. Скорины, \\ 246019 Гомель, Беларусь \\ e-mail: anton.shamyna@gmail.com
}

Поступила в редакцию 03.08.2017 г.

В приближении Релея-Ганса-Дебая решена задача о генерации гармоники суммарной частоты двумя плоскими эллиптически поляризованными электромагнитными волнами от поверхности диэлектрической частицы цилиндрической формы, покрытой тонким слоем, обладающим нелинейными оптическими свойствами. Формулы, описывающие поле суммарной частоты, представлены в тензорной и векторной формах для тензора нелинейной диэлектрической восприимчивости второго порядка, выбранного в общем виде, содержащем киральные компоненты. Получены выражения, описывающие поле суммарной частоты от торцов цилиндрической частицы, для случая нелинейного слоя, обладающего киральными свойствами. Трехмерные диаграммы направленности излучения суммарной частоты проанализированы для различных комбинаций параметров (углы падения, степени эллиптичности, ориентация эллипсов поляризации, размеры цилиндрической частицы). Обнаружены математические свойства функций пространственного распределения поля суммарной частоты, характеризующие симметрию диаграмм направленности.

DOI: $10.21883 /$ OS.2018.01.45366.176-17

\section{Введение}

Рост интереса к нелинейным оптическим эффектам второго порядка от поверхностей не прекращается на протяжении последних 20 лет. Это объясняется тем, что в дипольной модели, широко используемой в электродинамике, такие эффекты в объеме центросимметричных сред запрещены. Однако их можно наблюдать на границах раздела сред и в тонких пленках, что делает нелинейные оптические эффекты второго порядка уникальным инструментом для исследования свойств поверхностей.

Генерация суммарной частоты, благодаря большому количеству варьируемых параметров эксперимента (направления, частоты и поляризации падающих волн), является одним из самых перспективных нелинейных явлений для исследования поверхностей. На практике генерация суммарной частоты (и ее частный случай генерация второй гармоники) применяется для исследования капель воды [1,2], адсорбированных поверхностно активных веществ [3], биополимеров [4], коллоидных объектов [5], мембран биологических клеток [6], молекул ДНК [7].

Эффективное использование этого инструмента требует построения адекватной математической модели. На настоящий момент имеется три наиболее используемых модели: модель Релея-Ганса-Дебая [8-10], модель на основе точного решения задачи о рассеянии $[11,12]$ и модель Вентцеля-Крамерса-Бриллюэна [13]. Первая модель применима при малых размерах исследуемых систем и не учитывает рассеяние на границах раздела, однако для нее проще всего получить аналитическое решение. Вторая модель учитывает рассеяние, но ее ис- пользование затруднено для частиц произвольной формы, а также требует применения достаточно сложного математического аппарата. Третья модель лучше описывает нелинейные эффекты, чем первая, и применима для частиц произвольных размеров, но для нее можно получить только численное решение. В модели РелеяГанса-Дебая имеется ряд особенностей, не замеченных ранее, которым и посвящена настоящая статья.

\section{Дипольное приближение}

Пусть плоские электромагнитные волны в задаче задаются выражениями вида (у всех волн подразумевается временная часть $\exp (-i \omega t))$

$$
\mathbf{E}=E_{0} \mathbf{e} \exp (i \mathbf{k x}),
$$

где $E_{0}-$ это комплексная амплитуда волны, $\mathbf{e}-$ единичный комплексный вектор $\left(|\mathbf{e}|^{2}=\mathbf{e e}^{*}=1\right.$, где символ * означает комплексное сопряжение), характеризующий поляризацию (линейную, циркулярную или эллиптическую), $\mathbf{k}$ - волновой вектор.

За генерацию суммарной частоты в дипольной модели отвечает нелинейное слагаемое второго порядка в выражении для поляризации. Ее компоненты можно найти по формуле

$$
P_{i}^{(2)}=\chi_{i j k}^{(2)} E_{j}^{(1)} E_{k}^{(2)}
$$

где $\chi_{i j k}^{(2)}-$ тензор нелинейной диэлектрической восприимчивости второго порядка, $E_{j}^{(1)}, E_{k}^{(2)}-$ компоненты векторов напряженности электрического поля падающих волн, и подразумевается правило суммирования по повторяющимся индексам. 
Область применимости обобщенной модели РелеяГанса-Дебая ограничена выполнением следующих условий на частотах падающих волн:

$$
\left|\frac{n_{p}}{n_{m}}-1\right| \ll 1, \quad 4 \pi \frac{R}{\lambda}\left|\frac{n_{p}}{n_{m}}-1\right| \ll 1 .
$$

Здесь $n_{p}, n_{m}$ - показатели преломления диэлектрической частицы под нелинейным слоем и окружающей среды, $R$ - характерный размер частицы (для цилиндрической частицы - это ее радиус или длина), $\lambda-$ длина волны падающего излучения.

Введем сферическую систему координат $(r, \theta, \varphi)$ с базисными векторами $\left(\mathbf{e}_{r}, \mathbf{e}_{\theta}, \mathbf{e}_{\varphi}\right)$ и декартову систему координат $(x, y, z)$ с базисными векторами $\left(\mathbf{e}_{x}, \mathbf{e}_{y}, \mathbf{e}_{z}\right)$. Здесь и далее будем использовать гауссову систему единиц. Тогда методом функции Грина можно получить выражения для векторов магнитной индукции и электрической напряженности поля гармоники суммарной частоты в точке $\mathbf{x}$ (в дальней зоне):

$$
\mathbf{B}(\mathbf{x})=\mu k^{2} \sqrt{\varepsilon \mu} \frac{e^{i k \sqrt{\varepsilon \mu} r}}{r}\left[\mathbf{e}_{r} \times \int_{V} \exp \left(-i \mathbf{k} \mathbf{x}^{\prime}\right) \mathbf{P}\left(\mathbf{x}^{\prime}\right) d^{3} \mathbf{x}^{\prime}\right],
$$

$$
\mathbf{E}(\mathbf{x})=\mu k^{2} \frac{e^{i k \sqrt{\varepsilon \mu} r}}{r}\left(1-\mathbf{e}_{r} \otimes \mathbf{e}_{r}\right) \int_{V} \exp \left(-i \mathbf{k} \mathbf{x}^{\prime}\right) \mathbf{P}\left(\mathbf{x}^{\prime}\right) d^{3} \mathbf{x},
$$

где конструкция $[\mathbf{a} \times \mathbf{b}]$ означает векторное произведение, а символ $\otimes$ означает тензорное произведение векторов. Вектор $\mathbf{k}=k \sqrt{\varepsilon \mu} \mathbf{e}_{r}, k=\omega / c-$ модуль волнового вектора генерируемой волны в вакууме, а $\varepsilon, \mu-$ диэлектрическая и магнитная проницаемости окружающей среды на суммарной частоте $\omega, r=|\mathbf{x}|-$ расстояние до точки наблюдения, интегрирование ведется по всему объему $V$ нелинейного слоя.

\section{Генерация гармоники суммарной частоты в обобщенной модели Релея-Ганса-Дебая}

\section{Боковая поверхность}

Пусть цилиндрическая частица покрыта нелинейным слоем только на боковой поверхности. Поместим ее в центр сферической системы координат так, чтобы ось частицы совпадала с осью $O z$, а центр частицы совпадал с началом координат (общая схема задачи изображена на рис. 1). Выражение (5) в применении к генерации суммарной частоты выглядит следующим образом:

$$
\begin{aligned}
\mathbf{E}_{\text {lat }}^{(12)}(\mathbf{x})= & \left(1-\mathbf{e}_{r} \otimes \mathbf{e}_{r}\right) \mu_{12} \frac{\left(\omega_{12}\right)^{2}}{c^{2}} \frac{\exp \left(i k_{12} r\right)}{r} \\
& \times \int_{V} \exp \left(-i \mathbf{k}^{(12)} \mathbf{x}^{\prime}\right) \mathbf{P}^{(2)}\left(\mathbf{x}^{\prime}\right) d^{3} \mathbf{x}^{\prime},
\end{aligned}
$$

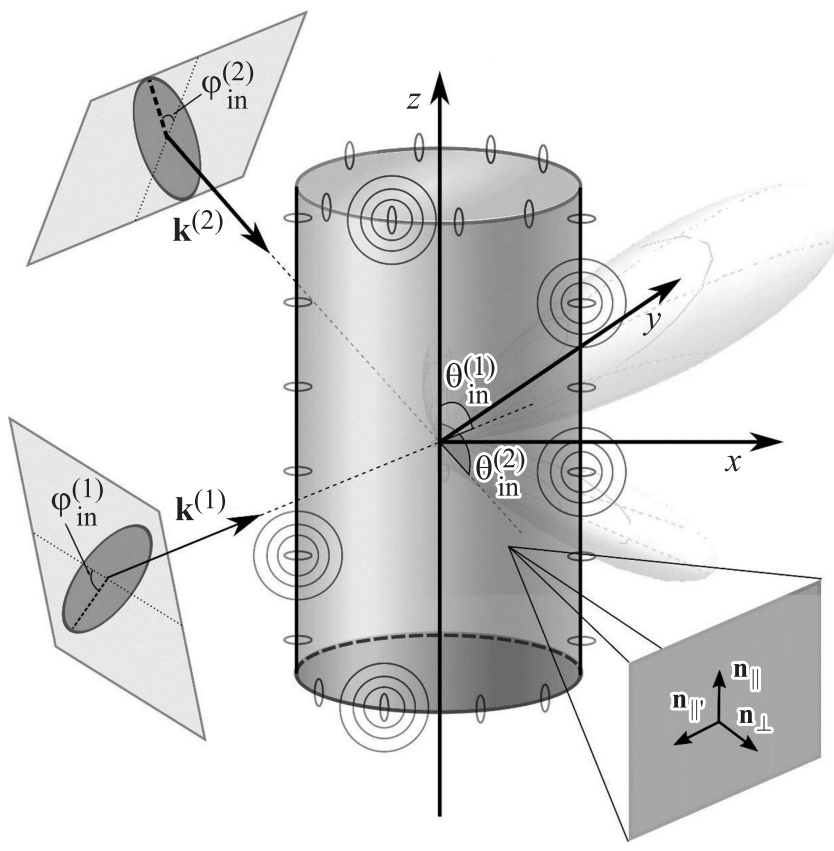

Рис. 1. Общая схема задачи о генерации излучения суммарной частоты от поверхности цилиндрической частицы.

где $\omega_{12}=\omega_{1}+\omega_{2}$ - циклическая частота генерируемых волн, $\mathbf{k}^{(12)}$ - волновой вектор гармоники суммарной частоты, $k_{12}=\left|\mathbf{k}^{(12)}\right|=\sqrt{\varepsilon_{12} \mu_{12}} \frac{\omega_{12}}{c}, \varepsilon_{12}, \mu_{12}$ - диэлектрическая и магнитная проницаемости среды на частоте $\omega_{12}$. Интегрирование ведется по всему объему $V$ нелинейного цилиндрического слоя на боковой поверхности.

В соответствии с (1) зададим векторы электрической напряженности падающих волн:

$$
\begin{aligned}
& \mathbf{E}^{(1)}(\mathbf{x})=E_{1} \mathbf{e}^{(1)} \exp \left(i \mathbf{k}^{(1)} \mathbf{x}\right), \\
& \mathbf{E}^{(2)}(\mathbf{x})=E_{2} \mathbf{e}^{(2)} \exp \left(i \mathbf{k}^{(2)} \mathbf{x}\right),
\end{aligned}
$$

где $\mathbf{k}^{(1)}$ и $\mathbf{k}^{(2)}$ - волновые векторы падающих волн, $E_{1}$ и $E_{2}-$ амплитуды падающих волн, а $\mathbf{e}^{(1)}$ и $\mathbf{e}^{(2)}-$ единичные векторы, характеризующие поляризацию падающих волн. Подставляя компоненты векторов из формулы (7) в (2), а затем в (6), получим

$$
\begin{aligned}
E_{\text {lat }, i}^{(12)}(\mathbf{x}) & =\mu_{12} \frac{\omega_{12}^{2}}{c^{2}} \frac{\exp \left(i k_{12} r\right)}{r} E_{1} E_{2} \\
& \times\left(\delta_{i m}-e_{r, i} e_{r, m}\right) e_{j}^{(1)} e_{k}^{(2)} \int_{V} \exp \left(i \mathbf{q} \mathbf{x}^{\prime}\right) \chi_{m j k}^{(2)}\left(\mathbf{x}^{\prime}\right) d^{3} \mathbf{x}^{\prime},
\end{aligned}
$$

где $\mathbf{q}$-вектор рассеяния, вычисляемый по формуле

$$
\mathbf{q}=\mathbf{k}^{(1)}+\mathbf{k}^{(2)}-\mathbf{k}^{(12)} \text {. }
$$

Вводя составляющие векторы рассеяния вдоль оси цилиндра и перпендикулярную ей

$$
\mathbf{q}_{\|}=\left(\mathbf{q e}_{z}\right) \mathbf{e}_{z}=q_{\|} \mathbf{e}_{z}, \quad \mathbf{q}_{\perp}=\mathbf{q}-\mathbf{q}_{\|}, \quad q_{\perp}=\left|\mathbf{q}_{\perp}\right|,
$$


получаем

$$
\begin{aligned}
& E_{\mathrm{lat}, i}^{(12)}(\mathbf{x})=\mu_{12} \frac{\omega_{12}^{2}}{c^{2}} \frac{\exp \left(i k_{12} r\right)}{r} E_{1} E_{2} \\
& \quad \times\left(\delta_{i m}-e_{r, i} e_{r, m}\right) e_{j}^{(1)} e_{k}^{(2)} \int_{a}^{a+d_{0}} r^{\prime} d r^{\prime} \int_{-h / 2}^{h / 2} \exp \left(i q_{\|} z^{\prime}\right) d z^{\prime}
\end{aligned}
$$$$
\times \int_{0}^{2 \pi} \exp \left(i a \mathbf{q}_{\perp} \mathbf{n}\right) \chi_{m j k}^{(2)}(\mathbf{n}) d \varphi^{\prime},
$$

где $a-$ радиус основания цилиндрической частицы, покрытой нелинейным слоем, $d_{0}-$ толщина слоя $\left(d_{0} \ll a\right), h$ - это высота цилиндрической частицы $\left(d_{0} \ll h\right), \mathbf{n}-$ единичный вектор нормали к боковой поверхности. После вычисления интегралов по $r^{\prime}$ и $z^{\prime}$ формула принимает вид

$$
\begin{aligned}
E_{\mathrm{lat}, i}^{(12)}(\mathbf{x})= & \mu_{12} \frac{\omega_{12}^{2}}{c^{2}} \frac{\exp \left(i k_{12} r\right)}{r} d_{0} a h E_{1} E_{2} \\
& \times\left(\delta_{i m}-e_{r, i} e_{r, m}\right) e_{j}^{(1)} e_{k}^{(2)} \frac{\sin \left(q_{\|} h / 2\right)}{q_{\|} h / 2} \\
& \times \int_{0}^{2 \pi} \exp \left(i a \mathbf{q}_{\perp} \mathbf{n}\right) \chi_{m j k}^{(2)}(\mathbf{n}) d \varphi^{\prime} .
\end{aligned}
$$

Вычисление интеграла по углу $\varphi^{\prime}$ требует подстановки явного вида тензора нелинейной диэлектрической восприимчивости $\chi_{m j k}^{(2)}$. В самом общем случае он представляется формулой

$$
\begin{aligned}
\chi_{i j k}^{(2)} & =\chi_{1}^{(2)} n_{i} n_{j} n_{k}+\chi_{2}^{(2)} n_{i} \delta_{j k}+\chi_{3}^{(2)} n_{j} \delta_{k i}+\chi_{4}^{(2)} n_{k} \delta_{i j} \\
& +\chi_{5}^{(2)} n_{m} n_{i} \varepsilon_{m j k}+\chi_{6}^{(2)} n_{m} n_{k} \varepsilon_{i j m}+\chi_{7}^{(2)} n_{m} n_{j} \varepsilon_{i m k},
\end{aligned}
$$

где $n_{i}$ - компоненты вектора нормали $\mathbf{n}$ к поверхности, $\delta_{i j}$ - дельта-символ Кронекера, $\varepsilon_{i j k}$ - символ ЛевиЧивита. Здесь учтено выполнение свойств симметрии при поворотах и инверсии. Коэффициенты $\chi_{1-7}^{(2)}-$ независимые коэффициенты, характеризующие анизотропные свойства нелинейного слоя, причем последние три из них обусловлены киральными свойствами слоя, поэтому их называют киральными. Первые четыре являются некиральными коэффициентами. При необходимости их можно связать с другими используемыми в литературе коэффициентами анизотропии:

$$
\begin{gathered}
\chi_{1}^{(2)}=\chi_{\perp \perp \perp}^{(2)}-\chi_{\perp\|\|}^{(2)}-\chi_{\|\perp\|}^{(2)}-\chi_{\|\| \perp}^{(2)}, \\
\chi_{2}^{(2)}=\chi_{\perp\|\| \|}^{(2)}=\chi_{\perp\left\|^{\prime}\right\|^{\prime}}^{(2)}, \chi_{3}^{(2)}=\chi_{\|\perp\|}^{(2)}=\chi_{\left\|^{\prime} \perp\right\|^{\prime}}^{(2)}, \\
\chi_{4}^{(2)}=\chi_{\|\| \perp}^{(2)}=\chi_{\left\|^{\prime}\right\|^{\prime} \perp}^{(2)}, \chi_{5}^{(2)}=\chi_{\perp\|\|^{\prime}}^{(2)}=-\chi_{\perp\left\|^{\prime}\right\|}^{(2)}, \\
\chi_{6}^{(2)}=\chi_{\|\|^{\prime} \perp}^{(2)}=-\chi_{\left\|^{\prime}\right\| \perp}^{(2)}, \quad \chi_{7}^{(2)}=\chi_{\left\|^{\prime} \perp\right\|}^{(2)}=-\chi_{\|\perp\|^{\prime}}^{(2)},
\end{gathered}
$$

где $\perp,\|,\|^{\prime}$ характеризуют направления, задаваемые правой тройкой единичных перпендикулярных векторов $\mathbf{n}_{\perp}=\mathbf{n}, \mathbf{n}_{\|}, \mathbf{n}_{\|^{\prime}}$ (рис. 1).
Подставляя (13) в (12), получаем выражение, содержащее следующие интегралы (подробное вычисление в приложении A):

$$
\begin{gathered}
\int_{0}^{2 \pi} \exp \left(i a \mathbf{q}_{\perp} \mathbf{n}\right) n_{k} d \varphi^{\prime}=2 \pi i J_{1}\left(q_{\perp} a\right) v_{k}, \\
\int_{0}^{2 \pi} \exp \left(i a \mathbf{q}_{\perp} \mathbf{n}\right) n_{i} n_{j} d \varphi^{\prime}=2 \pi\left[-v_{i} v_{j} J_{2}\left(q_{\perp} a\right)\right. \\
\left.\quad+\left(\delta_{i j}-\delta_{i z} \delta_{j z}\right) \frac{1}{2}\left(J_{0}\left(q_{\perp} a\right)+J_{2}\left(q_{\perp} a\right)\right)\right], \quad(16) \\
\int_{0}^{2 \pi} \exp \left(i a \mathbf{q}_{\perp} \mathbf{n}\right) n_{i} n_{j} n_{k} d \varphi^{\prime} \\
=2 \pi i\left[-J_{3}\left(q_{\perp} a\right) v_{i} v_{j} v_{k}+\frac{1}{4}\left(J_{1}\left(q_{\perp} a\right)+J_{3}\left(q_{\perp} a\right)\right)\right. \\
\left.\times\left(v_{i}\left(\delta_{j k}-\delta_{j z} \delta_{k z}\right)+v_{j}\left(\delta_{k i}-\delta_{i z} \delta_{k z}\right)+v_{k}\left(\delta_{i j}-\delta_{i z} \delta_{j z}\right)\right)\right],
\end{gathered}
$$

где $J_{m}(z)$ - цилиндрические функции Бесселя порядка $m, v_{i}-$ компоненты единичного вектора вдоль $\mathbf{q}_{\perp}$, индексы $i, j, k$ могут принимать значения $x, y, z$, а все появления символа $i$ вне индексов означают мнимую единицу. Введем тензор эффективной восприимчивости $\mathrm{X}_{i j k}^{(12)}$, определяемый из следующей формулы:

$$
\begin{aligned}
\mathrm{X}_{i j k}^{(12)} & =\frac{1}{2 \pi h} \int_{-h / 2}^{h / 2} \exp \left(i q_{\|} z^{\prime}\right) d z^{\prime} \int_{0}^{2 \pi} \exp \left(i a \mathbf{q}_{\perp} \mathbf{n}\right) \chi_{m j k}^{(2)}(\mathbf{n}) d \varphi^{\prime} \\
= & \frac{\sin \left(q_{\|} h / 2\right)}{q_{\|} h / 2}\left(i \chi _ { 1 } ^ { ( 2 ) } \left\{-J_{3}\left(q_{\perp} a\right) v_{i} v_{j} v_{k}\right.\right. \\
& +\frac{1}{4}\left(J_{1}\left(q_{\perp} a\right)+J_{3}\left(q_{\perp} a\right)\right)\left[v_{i}\left(\delta_{j k}-\delta_{j z} \delta_{k z}\right)\right. \\
& \left.\left.+v_{j}\left(\delta_{k i}-\delta_{i z} \delta_{k z}\right)+v_{k}\left(\delta_{i j}-\delta_{i z} \delta_{j z}\right)\right]\right\}+i J_{1}\left(q_{\perp} a\right) \\
& \times\left(\chi_{2}^{(2)} v_{i} \delta_{j k}+\chi_{3}^{(2)} v_{j} \delta_{i k}+\chi_{4}^{(2)} v_{k} \delta_{i j}\right) \\
& +\frac{1}{2}\left(J_{0}\left(q_{\perp} a\right)+J_{2}\left(q_{\perp} a\right)\right)\left(\chi_{5}^{(2)}+\chi_{6}^{(2)}+\chi_{7}^{(2)}\right) \varepsilon_{i j k} \\
& -\frac{1}{2}\left(J_{0}\left(q_{\perp} a\right)+J_{2}\left(q_{\perp} a\right)\right) \\
& \times\left(\chi_{5}^{(2)} \varepsilon_{z j k} \delta_{i z}+\chi_{6}^{(2)} \varepsilon_{i j z} \delta_{k z}+\chi_{7}^{(2)} \varepsilon_{i z k} \delta_{j z}\right) \\
& \left.-J_{2}\left(q_{\perp} a\right)\left(\chi_{5}^{(2)} v_{i} v_{m} \varepsilon_{m j k}+\chi_{6}^{(2)} v_{k} v_{m} \varepsilon_{i j m}+\chi_{7}^{(2)} v_{j} v_{m} \varepsilon_{i m k}\right)\right) .
\end{aligned}
$$

Введем также вектор $\mathbf{f}_{\text {lat }}^{(12)}$, компоненты которого определяются по формуле $f_{\text {lat }, i}^{(12)}=\mathrm{X}_{i j k}^{(12)} e_{j}^{(1)} e_{k}^{(2)}$. Он может 
быть представлен как

$$
\begin{aligned}
& \mathbf{f}_{\text {lat }}^{(12)}=\frac{\sin \left(q_{\|} h / 2\right)}{q_{\|} h / 2}\left[i \chi _ { 1 } ^ { ( 2 ) } \left(-J_{3}\left(q_{\perp} a\right) v\left(v \mathbf{e}^{(1)}\right)\left(v \mathbf{e}^{(2)}\right)\right.\right. \\
& +\frac{1}{4}\left(J_{1}\left(q_{\perp} a\right)+J_{3}\left(q_{\perp} a\right)\right) \\
& \times\left\{v\left[\left(\mathbf{e}^{(1)} \mathbf{e}^{(2)}\right)-\left(\mathbf{e}^{(1)} \mathbf{e}_{z}\right)\left(\mathbf{e}^{(2)} \mathbf{e}_{z}\right)\right]+\left(v \mathbf{e}^{(1)}\right)\right. \\
& \left.\left.\times\left[\mathbf{e}^{(2)}-\mathbf{e}_{z}\left(\mathbf{e}_{z} \mathbf{e}^{(2)}\right)\right]+\left(v \mathbf{e}^{(2)}\right)\left[\mathbf{e}^{(1)}-\mathbf{e}_{z}\left(\mathbf{e}_{z} \mathbf{e}^{(1)}\right)\right]\right\}\right) \\
& +i J_{1}\left(q_{\perp} a\right)\left[\chi_{2}^{(2)} v\left(\mathbf{e}^{(1)} \mathbf{e}^{(2)}\right)+\chi_{3}^{(2)} \mathbf{e}^{(2)}\left(\boldsymbol{v} \mathbf{e}^{(1)}\right)\right. \\
& \left.+\chi_{4}^{(2)} \mathbf{e}^{(1)}\left(\boldsymbol{v} \mathbf{e}^{(2)}\right)\right]+\frac{1}{2}\left(J_{0}\left(q_{\perp} a\right)+J_{2}\left(q_{\perp} a\right)\right) \\
& \times\left(\chi_{5}^{(2)}+\chi_{6}^{(2)}+\chi_{7}^{(2)}\right)\left[\mathbf{e}^{(1)} \times \mathbf{e}^{(2)}\right]-\frac{1}{2}\left(J_{0}\left(q_{\perp} a\right)+J_{2}\left(q_{\perp} a\right)\right) \\
& \times\left(\chi_{5}^{(2)} \mathbf{e}_{z}\left(\mathbf{e}_{z}\left[\mathbf{e}^{(1)} \times \mathbf{e}^{(2)}\right]\right)+\chi_{6}^{(2)}\left[\mathbf{e}^{(1)} \times \mathbf{e}_{z}\right]\left(\mathbf{e}_{z} \mathbf{e}^{(2)}\right)\right. \\
& \left.+\chi_{7}^{(2)}\left[\mathbf{e}_{z} \times \mathbf{e}^{(2)}\right]\left(\mathbf{e}_{z} \mathbf{e}^{(1)}\right)\right)-J_{2}\left(q_{\perp} a\right)\left(\chi_{5}^{(2)} \boldsymbol{v}\left(\boldsymbol{v}\left[\mathbf{e}^{(1)} \times \mathbf{e}^{(2)}\right]\right)\right. \\
& \left.\left.+\chi_{6}^{(2)}\left[\mathbf{e}^{(1)} \times v\right]\left(\boldsymbol{v} \mathbf{e}^{(2)}\right)+\chi_{7}^{(2)}\left[\boldsymbol{v} \times \mathbf{e}^{(2)}\right]\left(v \mathbf{e}^{(1)}\right)\right)\right] .
\end{aligned}
$$

Тогда вектор напряженности электрического поля гармоники суммарной частоты запишется в виде

$\mathbf{E}_{\text {lat }}^{(12)}(\mathbf{x})=2 \pi \mu_{12} \frac{\omega_{12}^{2}}{c^{2}} \frac{\exp \left(i k_{12} r\right)}{r} d_{0} a h E_{1} E_{2}\left(1-\mathbf{e}_{r} \otimes \mathbf{e}_{r}\right) \mathbf{f}_{\text {lat }}^{(12)}$.

Согласно (19), существуют направления, в которых излучение суммарной частоты от боковой поверхности не наблюдается. Это происходит при выполнении равенства

$$
q_{\|} h=2 \pi m, \quad m \neq 0 \text { - целое }
$$

\section{Торцы цилиндра}

Пусть цилиндрическая частица покрыта нелинейным слоем только на торцах. Тогда воспользовавшись формулой (5), но интегрируя по торцевой поверхности цилиндра, можно найти соответствующее поле суммарной частоты:

$$
\begin{aligned}
& E_{\mathrm{be}, i}^{(12)}(\mathbf{x})=\mu_{12} \frac{\omega_{12}^{2}}{c^{2}} \frac{\exp \left(i k_{12} r\right)}{r} E_{1} E_{2}\left(\delta_{i m}-e_{r, i} e_{r, m}\right) e_{j}^{(1)} e_{k}^{(2)} \\
& \times\left[\int_{h / 2}^{h / 2+d_{0}} \exp \left(i q_{\|} z^{\prime}\right) \chi_{m j k}^{(2)}\left(z^{\prime}\right) d z^{\prime} \int_{0}^{a} r^{\prime} d r^{\prime}\right. \\
& \times \int_{0}^{2 \pi} \exp \left(i q_{\perp} r^{\prime} \cos \varphi^{\prime}\right) d \varphi^{\prime}+\int_{-h / 2-d_{0}}^{-h / 2} \exp \left(i q_{\|} z^{\prime}\right) \chi_{m j k}^{(2)}\left(z^{\prime}\right) d z^{\prime} \\
& \left.\times \int_{0}^{a} r^{\prime} d r^{\prime} \int_{0}^{2 \pi} \exp \left(i q_{\perp} r^{\prime} \cos \varphi^{\prime}\right) d \varphi^{\prime}\right],
\end{aligned}
$$

где первый интеграл в квадратных скобках позволяет получить поле от верхнего торца цилиндра, а второй от нижнего торца. Вычисляя каждый из интегралов в (22), получаем следующее выражение:

$$
\begin{aligned}
& E_{\mathrm{be}, i}^{(12)}(\mathbf{x})=\pi \mu_{12} \frac{\omega_{12}^{2}}{c^{2}} \frac{\exp \left(i k_{12} r\right)}{r} E_{1} E_{2} a^{2} d_{0} \\
& \quad \times\left(\delta_{i m}-e_{r, i} e_{r, m}\right) e_{j}^{(1)} e_{k}^{(2)}\left(J_{0}\left(q_{\perp} a\right)+J_{2}\left(q_{\perp} a\right)\right) \\
& \quad \times\left[\exp \left(i q_{\|} h / 2\right) \chi_{m j k}^{(2)}(h / 2)+\exp \left(-i q_{\|} h / 2\right) \chi_{m j k}^{(12)}(-h / 2)\right],
\end{aligned}
$$

где тензоры нелинейной диэлектрической восприимчивости $\chi_{m j k}^{(2)}( \pm h / 2)$ на верхнем и нижнем торцах выражаются через $e_{z, i}-$ компоненты единичного вектора вдоль оси $O z$ (для верхнего торца вектор нормали к поверхности $\mathbf{n}=\mathbf{e}_{z}$, для нижнего $\left.\mathbf{n}=-\mathbf{e}_{z}\right)$ :

$$
\begin{aligned}
\chi_{m j k}^{(2)}(h / 2) & =\chi_{1}^{(2)} e_{z, m} e_{z, j} e_{z, k}+\chi_{2}^{(2)} e_{z, m} \delta_{j k} \\
& +\chi_{3}^{(2)} e_{z, j} \delta_{k m}+\chi_{4}^{(2)} e_{z, k} \delta_{m j}+\chi_{5}^{(2)} e_{z, l} e_{z, m} \varepsilon_{l j k} \\
& +\chi_{6}^{(2)} e_{z, l} e_{z, k} \varepsilon_{m j l}+\chi_{7}^{(2)} e_{z, l} e_{z, j} \varepsilon_{m l k}, \\
\chi_{m j k}^{(2)}(-h / 2) & =-\left(\chi_{1}^{(2)} e_{z, m} e_{z, j} e_{z, k}+\chi_{2}^{(2)} e_{z, m} \delta_{j k}\right. \\
& \left.+\chi_{3}^{(2)} e_{z, j} \delta_{k m}+\chi_{4}^{(2)} e_{z, k} \delta_{m j}\right)+\chi_{5}^{(2)} e_{z, l} e_{z, m} \varepsilon_{l j k} \\
& +\chi_{6}^{(2)} e_{z, l} e_{z, k} \varepsilon_{m j l}+\chi_{7}^{(2)} e_{z, l} e_{z, j} \varepsilon_{m l k} .
\end{aligned}
$$

Формула (23) с учетом (24) принимает вид

$$
\begin{aligned}
& E_{\mathrm{be}, i}^{(12)}(\mathbf{x})=2 \pi \mu_{12} \frac{\omega_{12}^{2}}{c^{2}} \frac{\exp \left(i k_{12} r\right)}{r} E_{1} E_{2} a^{2} d_{0} \\
& \times\left(\delta_{i m}-e_{r, i} e_{r, m}\right) e_{j}^{(1)} e_{k}^{(2)}\left(J_{0}\left(q_{\perp} a\right)+J_{2}\left(q_{\perp} a\right)\right) \\
& \times\left[i \operatorname { s i n } ( q _ { \| } h / 2 ) \left(\chi_{1}^{(2)} e_{z, m} e_{z, j} e_{z, k}+\chi_{2}^{(2)} e_{z, m} \delta_{j k}\right.\right. \\
& \left.+\chi_{3}^{(2)} e_{z, j} \delta_{k m}+\chi_{4}^{(2)} e_{z, k} \delta_{m j}\right)+\cos \left(q_{\|} h / 2\right) \\
& \left.\times\left(\chi_{5}^{(2)} e_{z, l} e_{z, m} \varepsilon_{l j k}+\chi_{6}^{(2)} e_{z, l} e_{z, k} \varepsilon_{m j l}+\chi_{7}^{(2)} e_{z, l} e_{z, j} \varepsilon_{m l k}\right)\right] .
\end{aligned}
$$

Важно отметить, что как для торцов, так и для боковой поверхности киральная и некиральная части поля суммарной частоты отличаются по фазе на множитель 
$i=e^{i \pi / 2}$. В векторном виде формула запишется как

$$
\begin{aligned}
& \mathbf{E}_{\mathrm{be}}^{(12)}(\mathbf{x})=2 \pi \mu_{12} \frac{\omega_{12}^{2}}{c^{2}} \frac{\exp \left(i k_{12} r\right)}{r} E_{1} E_{2} a^{2} d_{0}\left(1-\mathbf{e}_{r} \otimes \mathbf{e}_{r}\right) \\
& \times\left\{( J _ { 0 } ( q _ { \perp } a ) + J _ { 2 } ( q _ { \perp } a ) ) \left[i \operatorname { s i n } ( q _ { \| } h / 2 ) \left(\chi_{1}^{(2)} \mathbf{e}_{z}\left(\mathbf{e}_{z} \mathbf{e}^{(1)}\right)\left(\mathbf{e}_{z} \mathbf{e}^{(2)}\right)\right.\right.\right. \\
& \left.+\chi_{1}^{(2)} \mathbf{e}_{z}\left(\mathbf{e}^{(1)} \mathbf{e}^{(2)}\right)+\chi_{3}^{(2)} \mathbf{e}^{(2)}\left(\mathbf{e}_{z} \mathbf{e}^{(1)}\right)+\chi_{4}^{(2)} \mathbf{e}^{(1)}\left(\mathbf{e}_{z} \mathbf{e}^{(2)}\right)\right) \\
& +\cos \left(q_{\|} h / 2\right)\left(\chi_{5}^{(2)} \mathbf{e}_{z}\left(\mathbf{e}_{z}\left[\mathbf{e}^{(1)} \times \mathbf{e}^{(2)}\right]\right)+\chi_{6}^{(2)}\left[\mathbf{e}^{(1)} \times \mathbf{e}_{z}\right]\right. \\
& \left.\left.\left.\times\left(\mathbf{e}_{z} \mathbf{e}^{(2)}\right)+\chi_{7}^{(2)}\left[\mathbf{e}_{z} \times \mathbf{e}^{(2)}\right]\left(\mathbf{e}_{z} \mathbf{e}^{(1)}\right)\right)\right]\right\} \\
& =2 \pi \mu_{12} \frac{\omega_{12}^{2}}{c^{2}} \frac{\exp \left(i k_{12} r\right)}{r} E_{1} E_{2} a^{2} d_{0}\left(1-\mathbf{e}_{r} \otimes \mathbf{e}_{r}\right)\left\{\mathbf{f}_{\mathrm{be}}^{(12)}\right\},
\end{aligned}
$$

где $\mathbf{f}_{\mathrm{be}}^{(12)}$ - вспомогательный вектор, характеризующий пространственное распределение поля суммарной частоты, генерируемого от торцов цилиндрической частицы.

Согласно (26), максимум излучения от торцов цилиндра должен наблюдаться в направлении, для которого $q_{\perp}=0$. С другой стороны, в направлениях, для которых $J_{0}\left(q_{\perp} a\right)+J_{2}\left(q_{\perp} a\right)=0$, излучение суммарной частоты будет отсутствовать. Для некирального слоя $\left(\chi_{5-7}^{(2)}=0\right)$ излучение будет отсутствовать также в направлениях $q_{\|} h=2 \pi m, m-$ целое. Для слоя, обладающего только киральными свойствами $\left(\chi_{1-4}^{(2)}=0\right)$, условие отсутствия генерации запишется как $q_{\|} h=\pi(2 m+1), m$ - целое.

Поле, генерируемое от цилиндра, поверхность которого полностью покрыта нелинейным слоем, может быть получено как суперпозиция поля от боковой поверхности и от торцевых поверхностей цилиндра:

$$
\begin{aligned}
& \mathbf{E}^{(12)}(\mathbf{x})=\mathbf{E}_{\text {lat }}^{(12)}(\mathbf{x})+\mathbf{E}_{\mathrm{be}}^{(12)}(\mathbf{x}) \\
& \quad=2 \pi \mu_{12} \frac{\omega_{12}^{2}}{c^{2}} \frac{\exp \left(i k_{12} r\right)}{r} E_{1} E_{2} a h d_{0}\left(1-\mathbf{e}_{r} \otimes \mathbf{e}_{r}\right) \mathbf{f}^{(12)},
\end{aligned}
$$

где

$$
\mathbf{f}^{(12)}=\mathbf{f}_{\mathrm{lat}}^{(12)}+\frac{a}{h} \mathbf{f}_{\mathrm{be}}^{(12)}
$$

- вспомогательный вектор, характеризующий пространственное распределение поля суммарной частоты, генерируемого всей поверхностью цилиндра.

Зная напряженность электрического поля $\mathbf{E}(\mathbf{x})$ плоской электромагнитной волны и пользуясь уравнениями Максвелла, можно найти выражение для напряженности $\mathbf{H}(\mathbf{x})$ магнитного поля этой волны:

$$
\mathbf{H}(\mathbf{x})=\frac{n}{\mu}\left[\mathbf{e}_{r} \times \mathbf{E}(\mathbf{x})\right],
$$

где $n-$ показатель преломления среды, $\mu$ - магнитная проницаемость среды. Зная $\mathbf{H}(\mathbf{x})$ и $\mathbf{E}(\mathbf{x})$, можно найти модуль вектора Умова-Пойнтинга:

$$
\mathbf{S}(\mathbf{x})=\frac{1}{2} \frac{c}{4 \pi} \operatorname{Re}\left[\mathbf{E}(\mathbf{x}) \times \mathbf{H}^{*}(\mathbf{x})\right],|\mathbf{S}(\mathbf{x})|=\frac{c}{8 \pi} \frac{n}{\mu}|\mathbf{E}(\mathbf{x})|^{2} .
$$

Проводя аналогичные (6)-(26) рассуждения, но отталкиваясь от (4), можно получить выражение для напряженности магнитного поля генерируемой волны $\mathbf{H}^{(12)}(\mathbf{x})$. Этого же результата можно достичь, подставляя выражение для напряженности электрического поля генерируемой волны (27) в (29):

$$
\mathbf{H}^{(12)}(\mathbf{x})=\frac{n_{12}}{\mu_{12}}\left[\mathbf{e}_{r} \times \mathbf{E}^{(12)}(\mathbf{x})\right],
$$

где $n_{12}$ - показатель преломления среды на частоте $\omega_{12}$. Воспользовавшись формулами (27) и (30), запишем также выражение для радиальной составляющей вектора Умова-Пойнтинга в дальней зоне:

$$
S_{r}^{(12)}(\mathbf{x})=\frac{c}{8 \pi} \frac{n_{12}}{\mu_{12}}\left|\mathbf{E}^{(12)}(\mathbf{x})\right|^{2} .
$$

\section{Предельные формы решения}

Чтобы проанализировать поведение вектора $\mathbf{f}^{(12)}(\theta, \varphi)$ при больших и малых значениях размеров цилиндра, нам понадобятся приближенные выражения для цилиндрических функций Бесселя:

$$
\begin{gathered}
J_{m}(z)=\frac{z^{m}}{2^{m} m !}, \quad z \ll 1, \\
J_{m}(z)=\sqrt{\frac{2}{\pi z}} \cos \left(z-\frac{m \pi}{2}-\frac{\pi}{4}\right) \\
-\frac{4 m^{2}-1}{4 \sqrt{2 \pi z^{3}}} \sin \left(z-\frac{m \pi}{2}-\frac{\pi}{4}\right), \quad z \gg 1 .
\end{gathered}
$$

Тогда при малых значениях радиуса основания цилиндрической частицы $\left(q_{\perp} a \ll 1\right)$

$$
\begin{aligned}
& \mathbf{f}_{\text {lat }}^{(12)}=\frac{\sin \left(q_{\|} h / 2\right)}{q_{\|} h / 2}\left(i \chi _ { 1 } ^ { ( 2 ) } \frac { q _ { \perp } a } { 8 } \left\{v \left[\left(\mathbf{e}^{(1)} \mathbf{e}^{(2)}\right)-\left(\mathbf{e}^{(1)} \mathbf{e}_{z}\right)\right.\right.\right. \\
& \left.\times\left(\mathbf{e}^{(2)} \mathbf{e}_{z}\right)\right]+\left(v \mathbf{e}^{(1)}\right)\left[\mathbf{e}^{(2)}-\mathbf{e}_{z}\left(\mathbf{e}_{z} \mathbf{e}^{(2)}\right)\right]+\left(v \mathbf{e}^{(2)}\right) \\
& \left.\times\left[\mathbf{e}^{(1)}-\mathbf{e}_{z}\left(\mathbf{e}_{z} \mathbf{e}^{(1)}\right)\right]\right\}+i \frac{q_{\perp} a}{2}\left[\chi_{2}^{(2)} \boldsymbol{v}\left(\mathbf{e}^{(1)} \mathbf{e}^{(2)}\right)\right. \\
& \left.+\chi_{3}^{(2)} \mathbf{e}^{(2)}\left(\boldsymbol{v} \mathbf{e}^{(1)}\right)+\chi_{4}^{(2)} \mathbf{e}^{(1)}\left(\boldsymbol{v} \mathbf{e}^{(2)}\right)\right]+\frac{1}{2}\left(\chi_{5}^{(2)}+\chi_{6}^{(2)}+\chi_{7}^{(2)}\right) \\
& \times\left[\mathbf{e}^{(1)} \times \mathbf{e}^{(2)}\right]-\frac{1}{2}\left(\chi_{5}^{(2)} \mathbf{e}_{z}\left(\mathbf{e}_{z}\left[\mathbf{e}^{(1)} \times \mathbf{e}^{(2)}\right]\right)\right. \\
& \left.\left.+\chi_{6}^{(2)}\left[\mathbf{e}^{(1)} \times \mathbf{e}_{z}\right]\left(\mathbf{e}_{z} \mathbf{e}^{(2)}\right)+\chi_{7}^{(2)}\left[\mathbf{e}_{z} \times \mathbf{e}^{(2)}\right]\left(\mathbf{e}_{z} \mathbf{e}^{(1)}\right)\right)\right),
\end{aligned}
$$


а при больших значениях радиуса основания цилиндрической частицы $\left(q_{\perp} a \gg 1\right)$

$$
\begin{aligned}
\mathbf{f}_{\text {lat }}^{(12)}= & \frac{\sin \left(q_{\|} h / 2\right)}{\left.q_{\|} h / 2\right)}\left\{\frac{i \sqrt{2} \sin \left(q_{\perp} a-\pi / 4\right)}{\sqrt{\pi q_{\perp} a}}\right. \\
& \times\left[\chi_{1}^{(2)} \boldsymbol{v}\left(\boldsymbol{v} \mathbf{e}^{(1)}\right)\left(\boldsymbol{v} \mathbf{e}^{(2)}\right)+\chi_{2}^{(2)} \boldsymbol{v}\left(\mathbf{e}^{(1)} \mathbf{e}^{(2)}\right)\right. \\
& \left.+\chi_{3}^{(2)} \mathbf{e}^{(2)}\left(\boldsymbol{v} \mathbf{e}^{(1)}\right)+\chi_{4}^{(2)} \mathbf{e}^{(1)}\left(\boldsymbol{v} \mathbf{e}^{(2)}\right)\right] \\
& +\frac{\sqrt{2} \cos \left(q_{\perp} a-\pi / 4\right)}{\sqrt{\pi q_{\perp} a}}\left[\chi_{5}^{(2)} v\left(\boldsymbol{v}\left[\mathbf{e}^{(1)} \times \mathbf{e}^{(2)}\right]\right)\right. \\
& +\chi_{6}^{(2)}\left[\mathbf{e}^{(1)} \times \boldsymbol{v}\right]\left(\boldsymbol{v} \mathbf{e}^{(2)}\right) \\
& \left.\left.+\chi_{7}^{(2)}\left[\boldsymbol{v} \times \mathbf{e}^{(2)}\right]\left(\boldsymbol{v} \mathbf{e}^{(1)}\right)\right]\right\} .
\end{aligned}
$$

Анализируя выражение (34) для вектора $\mathbf{f}_{\text {lat }}^{(12)}$ при малых значениях $a$, можно прийти к выводу, что влияние киральных коэффициентов $\chi_{5-7}^{(2)}$ на генерацию суммарной гармоники преобладает над влиянием некиральных коэффициентов $\chi_{1-4}^{(2)}$. При этом мощность генерируемых волн пропорциональна $a^{2} h^{2}$. Если нелинейный слой не обладает киральными свойствами $\left(\chi_{5-7}^{(2)}=0\right)$, то $S_{\text {lat }, r}^{(12)} \sim a^{4} h^{2}$.

Теперь рассмотрим влияние высоты $h$ на вектор $\mathbf{f}_{\text {lat }}^{(12)}$. При малых $h\left(\left|q_{\|} h\right| \ll 1\right)$ коэффициент, зависящий от $h$, упрощается:

$$
\frac{\sin \left(q_{\|} h / 2\right)}{q_{\|} h / 2} \approx 1,
$$

и пространственное распределение генерируемого излучения в этом случае не зависит от $q_{\|}$. Если же $h$ принимает большие значения $\left(\left|q_{\|} h\right| \gg 1\right)$, то

$$
\frac{\sin \left(q_{\|} h / 2\right)}{q_{\|} h / 2} \rightarrow \frac{2 \pi}{h} \delta\left(q_{\|}\right),
$$

где $\delta\left(q_{\|}\right)$- дельта-функция Дирака. Значит, излучение будет фиксироваться преимущественно в направлениях, для которых $q_{\|} \rightarrow 0$.

Согласно (34), в направлениях, где $q_{\perp}=0$, некиральные коэффициенты не вносят вклад в генерацию суммарной частоты. В этом случае за генерацию отвечают киральные коэффициенты. Регистрация излучения суммарной частоты позволит оценить величину киральных коэффициентов в тензоре нелинейной диэлектрической восприимчивости. При больших значениях $a$, согласно (35), можно наблюдать множество локальных максимумов излучения суммарной частоты. Причем их амплитуда убывает с отклонением от направления, для которого $q_{\perp}=0$.
Рассмотрим поведение функций, входящих в формулу для $\mathbf{f}_{\text {lat }}^{(12)}$, при больших и малых размерах цилиндра:

$$
\begin{array}{rlrl}
J_{0}\left(q_{\perp} a\right)+J_{2}\left(q_{\perp} a\right)=1, & q_{\perp} a \ll 1, \\
J_{0}\left(q_{\perp} a\right)+J_{2}\left(q_{\perp} a\right)=\frac{4}{\sqrt{2 \pi\left(q_{\perp} a\right)^{3}}} \sin \left(q_{\perp} a-\frac{\pi}{4}\right), & q_{\perp} a \gg 1, \\
\sin \left(q_{\|} h / 2\right)=q_{\|} h / 2, & \cos \left(q_{\|} h / 2\right)=1, & \left|q_{\|} h\right| \ll 1 .
\end{array}
$$

Следовательно, при малых высотах цилиндрической частицы $h$ в генерацию поля суммарной частоты от его торцов решающий вклад вносят киральные коэффициенты $\chi_{5-7}^{(2)}$, а мощность излучения $S_{\mathrm{be}, r}^{(12)} \sim a^{4}$. Если нелинейный оптический слой не обладает киральными свойствами $\left(\chi_{5-7}^{(2)}=0\right)$, то $S_{\mathrm{be}, r}^{(12)} \sim a^{4} h^{2}$.

И для торцов, и для боковой поверхности было обнаружено, что излучение, обусловленное киральными компонентами тензора нелинейной восприимчивости, преобладает над излучением, обусловленным некиральными компонентами, при малых размерах цилиндрической частицы. Качественно это можно объяснить следующим образом. Противоположные точки цилиндра, векторы нормали в которых антипараллельны, генерируют электромагнитные волны, отличающиеся по фазе на $\pi$, если поверхность не обладает киральными свойствами $\left(\chi_{1-4}^{(2)} \neq 0, \chi_{5-7}^{(2)}=0\right)$, так как в этом случае $\chi_{i j k}^{(2)}(-\mathbf{n})=-\chi_{i j k}^{(2)}(\mathbf{n})$. Если поверхность обладает только киральными свойствами $\left(\chi_{1-4}^{(2)}=0, \chi_{5-7}^{(2)} \neq 0\right)$, то генерируются электромагнитные волны одной фазы, так как $\chi_{i j k}^{(2)}(-\mathbf{n})=\chi_{i j k}^{(2)}(\mathbf{n})$. Таким образом, при малых размерах цилиндрической частицы излучение, обусловленное некиральными компонентами, значительно ослабляется, а излучение, обусловленное киральными компонентами, усиливается. Эти же рассуждения верны и для сферических частиц.

\section{Анализ решения}

\section{Явный вид векторов в задаче}

Анализ полученных формул удобнее проводить графически. Наглядное представление пространственного распределения поля суммарной частоты возможно с помощью трехмерных диаграмм направленности. Для их построения необходимо задать явный вид всех векторов, используемых в решении. Введем цилиндрическую систему координат $(\rho, \varphi, z)$ с базисными векторами $\left(\mathbf{e}_{\rho}, \mathbf{e}_{\varphi}, \mathbf{e}_{z}\right)$. Пусть ось $O z$ направлена вверх, а ось $O x$ вправо вдоль вектора $\mathbf{k}_{\perp}^{(1+2)}=\left(1-\mathbf{e}_{z} \otimes \mathbf{e}_{z}\right)\left(\mathbf{k}^{(1)}+\mathbf{k}^{(2)}\right)$. Введем угол раскрытия $\gamma$, как угол между составляющими векторов $\mathbf{k}^{(1)}$ и $\mathbf{k}^{(2)}$, перпендикулярными вектору $\mathbf{e}_{z}$, т. е. между $\left(1-\mathbf{e}_{z} \otimes \mathbf{e}_{z}\right) \mathbf{k}^{(1)}$ и $\left(1-\mathbf{e}_{z} \otimes \mathbf{e}_{z}\right) \mathbf{k}^{(2)}$. Схема векторов в задаче представлена на рис. 2. Тогда углы 
между последними векторами и осью $O x$ равны соответственно

$$
\begin{aligned}
& \gamma_{1}=\arccos \frac{\left(k_{1} \sin \theta_{\text {in }}^{(1)}\right)+\left(k_{2} \sin \theta_{\text {in }}^{(2)}\right) \cos \gamma}{\sqrt{\left(k_{1} \sin \theta_{\text {in }}^{(1)}\right)^{2}+\left(k_{2} \sin \theta_{\text {in }}^{(2)}\right)^{2}+2\left(k_{1} \sin \theta_{\text {in }}^{(1)}\right)\left(k_{2} \sin \theta_{\text {in }}^{(2)}\right) \cos \gamma}}, \\
& \gamma_{2}=\arccos \frac{\left(k_{2} \sin \theta_{\text {in }}^{(2)}\right)+\left(k_{1} \sin \theta_{\text {in }}^{(1)}\right) \cos \gamma}{\sqrt{\left(k_{1} \sin \theta_{\text {in }}^{(1)}\right)^{2}+\left(k_{2} \sin \theta_{\text {in }}^{(2)}\right)^{2}+2\left(k_{1} \sin \theta_{\text {in }}^{(1)}\right)\left(k_{2} \sin \theta_{\text {in }}^{(2)}\right) \cos \gamma}},
\end{aligned}
$$

где $k_{1}=\left|\mathbf{k}^{(1)}\right|$ и $k_{2}=\left|\mathbf{k}^{(2)}\right|$ — модули волновых векторов падающих волн, а $\theta_{\text {in }}^{(1)}$ и $\theta_{\text {in }}^{(2)}$ - углы между осью $O z$ и волновыми векторами, при этом $\gamma=\gamma_{1}+\gamma_{2}$. Если $\left(k_{1} \sin \theta_{\text {in }}^{(1)}\right)^{2}+\left(k_{2} \sin \theta_{\text {in }}^{(2)}\right)^{2}+2\left(k_{1} \sin \theta_{\text {in }}^{(1)}\right)\left(k_{2} \sin \theta_{\text {in }}^{(2)}\right) \cos \gamma=0$, то $\gamma_{1}=0, \gamma_{2}=\gamma-\gamma_{1}$

С использованием этих обозначений выражения для волновых векторов и векторов поляризации падающих волн запишутся в виде

$$
\begin{aligned}
& \mathbf{k}^{(1)}=k_{1} \cos \theta_{\text {in }}^{(1)} \mathbf{e}_{z}+k_{1} \sin \theta_{\text {in }}^{(1)}\left(\sin \gamma_{1} \mathbf{e}_{y}+\cos \gamma_{1} \mathbf{e}_{x}\right), \\
& \mathbf{k}^{(2)}=k_{2} \cos \theta_{\text {in }}^{(2)} \mathbf{e}_{z}+k_{2} \sin \theta_{\text {in }}^{(2)}\left(-\sin \gamma_{2} \mathbf{e}_{y}+\cos \gamma_{2} \mathbf{e}_{x}\right), \\
& \mathbf{e}^{(1)}=\frac{-\sin \gamma_{1}\left(-i \sigma_{1} \cos \varphi_{\text {in }}^{(1)}-\sin \varphi_{\text {in }}^{(1)}\right)+\cos \gamma_{1} \cos \theta_{\text {in }}^{(1)}\left(-\cos \varphi_{\text {in }}^{(1)}+i \sigma_{1} \sin \varphi_{\text {in }}^{(1)}\right)}{\sqrt{1+\sigma_{1}^{2}}} \mathbf{e}_{x} \\
& +\frac{\cos \gamma_{1}\left(-i \sigma_{1} \cos \varphi_{\mathrm{in}}^{(1)}-\sin \varphi_{\mathrm{in}}^{(1)}\right)+\sin \gamma_{1} \cos \theta_{\mathrm{in}}^{(1)}\left(-\cos \varphi_{\mathrm{in}}^{(1)}+i \sigma_{1} \sin \varphi_{\mathrm{in}}^{(1)}\right)}{\sqrt{1+\sigma_{1}^{2}}} \mathbf{e}_{y} \\
& -\sin \theta_{\text {in }}^{(1)} \frac{-\cos \varphi_{\text {in }}^{(1)}+i \sigma_{1} \sin \varphi_{\text {in }}^{(1)}}{{\sqrt{1+\sigma_{1}}}^{2}} \mathbf{e}_{z}, \\
& \mathbf{e}^{(2)}=\frac{\sin \gamma_{2}\left(-i \sigma_{2} \cos \varphi_{\text {in }}^{(2)}-\sin \varphi_{\text {in }}^{(2)}\right)+\cos \gamma_{2} \cos \theta_{\text {in }}^{(2)}\left(-\cos \varphi_{\text {in }}^{(2)}+i \sigma_{2} \sin \varphi_{\text {in }}^{(2)}\right)}{\sqrt{1+\sigma_{2}^{2}}} \mathbf{e}_{x} \\
& +\frac{\cos \gamma_{2}\left(-i \sigma_{2} \cos \varphi_{\mathrm{in}}^{(2)}-\sin \varphi_{\mathrm{in}}^{(2)}\right)-\sin \gamma_{2} \cos \theta_{\mathrm{in}}^{(2)}\left(-\cos \varphi_{\mathrm{in}}^{(2)}+i \sigma_{2} \sin \varphi_{\mathrm{in}}^{(2)}\right)}{\sqrt{1+\sigma_{2}^{2}}} \mathbf{e}_{y} \\
& -\sin \theta_{\mathrm{in}}^{(2)} \frac{-\cos \varphi_{\mathrm{in}}^{(2)}+i \sigma_{2} \sin \varphi_{\mathrm{in}}^{(2)}}{{\sqrt{1+\sigma_{2}}}^{2}} \mathbf{e}_{z},
\end{aligned}
$$

где $\sigma_{1}, \sigma_{2}$ - коэффициенты, характеризующие степени эллиптичности падающих волн $(\sigma>0 / \sigma<0 / \sigma=0-$ правая/левая/линейная поляризация) и по модулю равные отношению длины меньшей полуоси эллипса поляризации к длине большей полуоси, $\varphi_{\text {in }}^{(1)}\left(\varphi_{\text {in }}^{(2)}\right)-$ угол между большей полуосью эллипса поляризации первой (второй) падающей волны и плоскостью, содержащей волновой вектор $\mathbf{k}^{(1)}\left(\mathbf{k}^{(2)}\right)$ и ось $O z$.

Воспользовавшись выражениями (40), запишем выражение для перпендикулярной составляющей вектора рассеяния:

$$
\begin{aligned}
\mathbf{q}_{\perp} & =\left(1-\mathbf{e}_{z} \otimes \mathbf{e}_{z}\right)\left(\mathbf{k}^{(1)}+\mathbf{k}^{(2)}-\mathbf{k}^{(12)}\right) \\
& =-\left[\left(k_{12} \sin \theta-\xi \cos \varphi\right) \mathbf{e}_{\rho}+\xi \sin \varphi \mathbf{e}_{\varphi}\right], \\
q_{\perp}=\left|\mathbf{q}_{\perp}\right| & =\sqrt{\xi^{2}-2 \xi k_{12} \sin \theta \cos \varphi+\left(k_{12} \sin \theta\right)^{2}} .
\end{aligned}
$$

Вспомогательная величина $\xi$ равна модулю составляющей суммы волновых векторов падающих волн, перпен- 

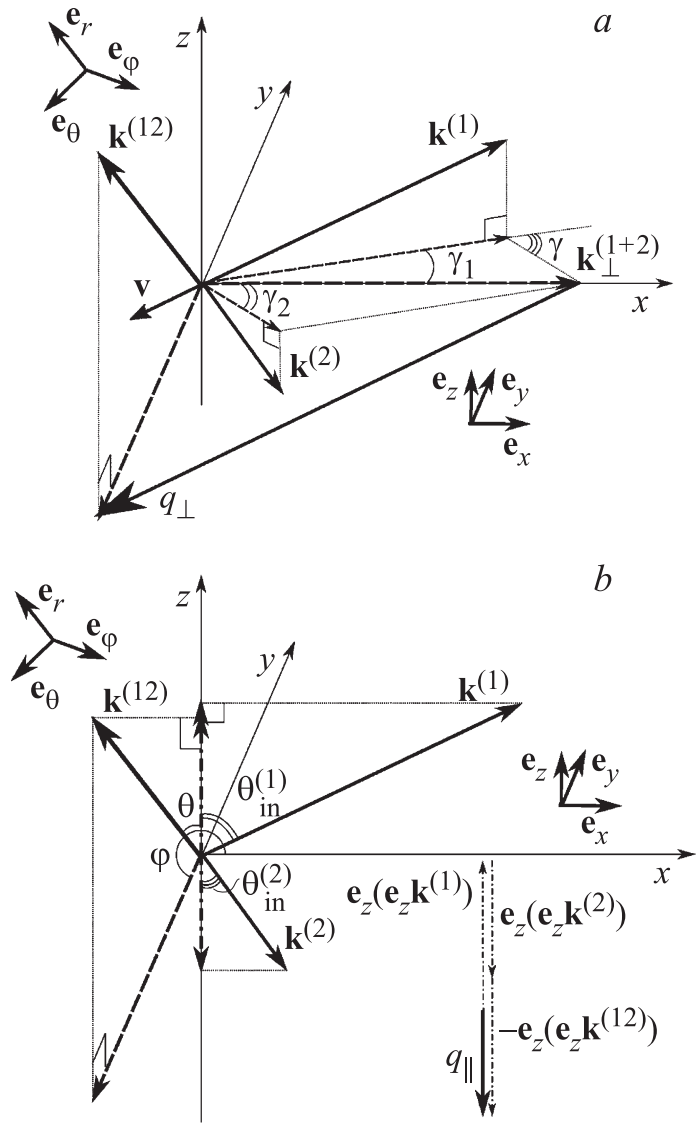

Рис. 2. Схема векторов, используемых в задаче: (a) векторы, необходимые для получения $\mathbf{q}_{\perp},(b)$ векторы, необходимые для получения $\mathbf{q}_{\|}$. Штриховыми линиями обозначены векторы в плоскости $O x y$. Штрихпунктирными линиями обозначены векторы вдоль оси $O z$.

дикулярной оси $O z$, и задается выражением

$$
\begin{aligned}
\xi & =\left|\left(1-\mathbf{e}_{z} \otimes \mathbf{e}_{z}\right)\left(\mathbf{k}^{(1)}+\mathbf{k}^{(2)}\right)\right| \\
& =\sqrt{\begin{array}{r}
\left(k_{1} \sin \theta_{\text {in }}^{(1)}\right)^{2}+\left(k_{2} \sin \theta_{\text {in }}^{(2)}\right)^{2}+ \\
+2\left(k_{1} \sin \theta_{\text {in }}^{(1)}\right)\left(k_{2} \sin \theta_{\text {in }}^{(2)}\right) \cos \gamma
\end{array}} .
\end{aligned}
$$

Степень дисперсии, характеризуемую коэффициентом

$$
\xi=\frac{k_{12}}{k_{1}+k_{2}}=\frac{n_{12} \omega_{12}}{n_{1} \omega_{1}+n_{2} \omega_{2}}
$$

$\left(n_{1}\right.$ и $n_{2}$ - показатели преломления на частотах $\omega_{1}$ и $\left.\omega_{2}\right)$, можно учесть, задавая значение модуля волнового вектора генерируемой волны $k_{12}$. Тогда единичный вектор вдоль $\mathbf{q}_{\perp}$ запишется в виде

$$
\begin{gathered}
\boldsymbol{v}=\frac{\mathbf{q}_{\perp}}{\left|\mathbf{q}_{\perp}\right|}=\frac{\left(-k_{12} \sin \theta+\xi \cos \varphi\right) \mathbf{e}_{\rho}-\xi \sin \varphi \mathbf{e}_{\varphi}}{\sqrt{\xi^{2}-2 \xi k_{12} \sin \theta \cos \varphi+\left(k_{12} \sin \theta\right)^{2}}}, \\
\boldsymbol{v}=0, \text { если } \xi^{2}-2 \xi k_{12} \sin \theta \cos \varphi+\left(k_{12} \sin \theta\right)^{2}=0 .
\end{gathered}
$$

Составляющая вектора q, параллельная оси $O z$, вычисляется по формуле

$$
\begin{gathered}
\mathbf{q}_{\|}=\mathbf{e}_{z}\left[\mathbf{e}_{z}\left(\mathbf{k}^{(1)}+\mathbf{k}^{(2)}-\mathbf{k}^{(12)}\right)\right] \\
=\mathbf{e}_{z}\left(k_{1} \cos \theta_{\text {in }}^{(1)}+k_{2} \cos \theta_{\text {in }}^{(2)}-k_{12} \cos \theta\right)=\mathbf{e}_{z} q_{\|}, \\
q_{\|}=\mathbf{e}_{z}\left(\mathbf{k}^{(1)}+\mathbf{k}^{(2)}-\mathbf{k}^{(12)}\right)=k_{1} \cos \theta_{\text {in }}^{(1)} \\
+k_{2} \cos \theta_{\text {in }}^{(2)}-k_{12} \cos \theta .
\end{gathered}
$$

\section{Свойства функций, характеризующих пространственное распределение поля суммарной частоты}

Явный вид зависимостей $\mathbf{f}^{(12)}(\theta, \varphi)$ и $S_{r}^{(12)}(\theta, \varphi)$ можно найти, подставив выражения (39)-(45) в формулы (19)-(32). При некоторых значениях параметров задачи (углов падения, ориентации эллипса поляризации, степени эллиптичности) у вектора $\mathbf{f}^{(12)}(\theta, \varphi)$ и функции проявляются математические свойства, характеризующие симметрию пространственного распределения генерируемого поля суммарной частоты (таблица). Их поиск позволяет проверить правильность решения на простых случаях. Рассмотрим некоторые из приведенных в таблице свойств.

Согласно второй строке при $\chi_{2}^{(2)} \neq 0, \quad \chi_{1}^{(2)}=0$, $\chi_{3-7}^{(2)}=0$ наблюдается зеркальная симметрия пространственного распределения мощности генерируемого излучения относительно плоскости $O x z$.

В строках с 5 по 11 представлены свойства, характерные для случаев, когда плоскости падения обеих волн совпадают с плоскостью $O x z$, т. е. угол раскрытия равен нулю $(\gamma=0)$. Так, согласно строке 5 , если волновые векторы падающих волн направлены вдоль оси $O x$, то наблюдается симметрия относительно поворота вокруг этой оси на $\pi$. Действительно, в этом случае симметрия схемы задачи определяет симметрию распределения генерируемого излучения.

Согласно шестой строке, если большие или меньшие полуоси эллипсов поляризации падающих волн совпадают с осью $O y$, то пространственное распределение мощности генерируемого излучения имеет зеркальную плоскость симметрии $O x z$. Это тоже можно объяснить симметрией задачи.

В строках с 12 по 20 рассмотрены свойства, наблюдаемые при направлениях волновых векторов падающих волн вдоль оси цилиндра. В строке 15 описан случай, когда на цилиндр вдоль его оси падают волны циркулярной поляризации. Тогда задача приобретает аксиальную симметрию, а мощность генерируемой волны суммарной частоты не зависит от азимутального угла наблюдения. Согласно строке 18 , когда все коэффициенты анизотропии $\chi_{1,3,4,6,7}^{(2)}$ равны нулю, распределение мощности излучения суммарной частоты имеет аксиальную симметрию независимо от поляризации падающих волн. 
Математические свойства функций, характеризующих пространственное распределение поля гармоники суммарной частоты

\begin{tabular}{|c|c|c|}
\hline № & Комбинация параметров & Свойства \\
\hline 1 & 2 & 3 \\
\hline 1 & $m_{1}, m_{2}$ - целые & $\begin{array}{l}\mathbf{f}^{(12)}\left(\theta+2 \pi m_{1}, \varphi+2 \pi m_{2}\right)=\mathbf{f}^{(12)}(\theta, \varphi), \\
\mathbf{f}^{(12)}(-\theta, \varphi)=-\left(1-2 \mathbf{e}_{r} \otimes \mathbf{e}_{r}\right) \mathbf{f}^{(12)}(\theta, \varphi+\pi), \\
S_{r}^{(12)}\left(\theta+2 \pi m_{1}, \varphi+2 \pi m_{2}\right)=S_{r}^{(12)}(\theta, \varphi) \\
S_{r}^{(12)}(-\theta, \varphi)=S_{r}^{(12)}(\theta, \varphi+\pi)\end{array}$ \\
\hline 2 & $\chi_{2}^{(2)} \neq 0, \chi_{1,3-7}^{(2)}=0$ & $\begin{array}{l}\mathbf{f}^{(12)}(\theta,-\varphi)=\left(1-2 \mathbf{e}_{\varphi} \otimes \mathbf{e}_{\varphi}\right) \mathbf{f}^{(12)}(\theta, \varphi) \\
S_{r}^{(12)}(\theta,-\varphi)=S_{r}^{(12)}(\theta, \varphi)\end{array}$ \\
\hline 3 & $\begin{array}{l}\chi_{2}^{(2)} \neq 0, \chi_{1,3-7}^{(2)}=0, \theta_{\mathrm{in}}^{(1)}=\theta_{\mathrm{in}}^{(2)}, \varphi_{\mathrm{in}}^{(2)}-\varphi_{\mathrm{in}}^{(1)}=\pi m_{1}, \\
\varphi_{\mathrm{in}}^{(1)}=\pi m_{2} / 2, \sigma_{1}=\sigma_{2}, m_{1}, m_{2}-\text { целые }\end{array}$ & $\operatorname{Re}\left[\mathbf{f}^{(12)}(\theta, \varphi)\right]=0$ \\
\hline 4 & $\begin{array}{l}\chi_{2}^{(2)} \neq 0, \chi_{1,3-7}^{(2)}=0, \theta_{\mathrm{in}}^{(1)}=\theta_{\mathrm{in}}^{(2)} \\
\varphi_{\mathrm{in}}^{(2)}-\varphi_{\mathrm{in}}^{(1)}=\pi m_{1} / 2, \sigma_{1}=\sigma_{2}=0, m_{1}-\text { целое }\end{array}$ & $\operatorname{Re}\left[\mathbf{f}^{(12)}(\theta, \varphi)\right]=0$ \\
\hline 5 & $\gamma=0, \pi, \theta_{\mathrm{in}}^{(1)}=\theta_{\mathrm{in}}^{(2)}=\pi / 2$ & $\begin{array}{l}\mathbf{f}^{(12)}(\theta,-\varphi)=-\left(1-2 \mathbf{e}_{r} \otimes \mathbf{e}_{r}\right) \mathbf{f}^{(12)}(\pi-\theta, \varphi) \\
\mathbf{e}_{\varphi} \mathbf{f}_{\text {lat }}^{(12)}(\theta,-\varphi)=-\mathbf{e}_{\varphi} \mathbf{f}_{\text {lat }}^{(12)}(\theta, \varphi), S_{r}^{(12)}(\theta,-\varphi)=S_{r}^{(12)}(\pi-\theta, \varphi)\end{array}$ \\
\hline 6 & $\begin{array}{l}\gamma=0, \varphi_{\text {in }}^{(1)}=\pi m_{1} / 2, \varphi_{\text {in }}^{(2)}=\pi m_{2} / 2, \\
m_{1}, m_{2} \text { - целые }\end{array}$ & $\begin{array}{l}\mathbf{f}^{(12)}(\theta,-\varphi)=\exp \left(i\left(m_{1}+m_{2}+1\right) \pi / 2\right)= \\
=\left[\left(1-2 \mathbf{e}_{\varphi} \otimes \mathbf{e}_{\varphi}\right) \mathbf{f}^{(12)}(\theta, \varphi) \exp \left(i\left(m_{1}+m_{2}+1\right) \pi / 2\right)\right] \\
S_{r}^{(12)}(\theta,-\varphi)=S_{r}^{(12)}(\theta, \varphi)\end{array}$ \\
\hline 7 & $\begin{array}{l}\gamma=0, \pi, \varphi_{\text {in }}^{(2)}-\varphi_{\text {in }}^{(1)}=\pi m_{1}, \varphi_{\text {in }}^{(1)}=m_{2} \pi+\pi / 2 \\
\sigma_{1}=\sigma_{2}=0, m_{1}, m_{2}-\text { целые }\end{array}$ & $\operatorname{Re}\left[\mathbf{e}_{\varphi} \mathbf{f}^{(12)}(\theta, \varphi)\right]=0$ \\
\hline 8 & $\begin{array}{l}\chi_{1,2,5}^{(2)} \neq 0, \chi_{3,4,6,7}^{(2)}=0 \\
\gamma=0, \pi, \theta_{\mathrm{in}}^{(2)}=\gamma+(-1)^{\gamma / \pi} \theta_{\mathrm{in}}^{(1)} \\
\varphi_{\mathrm{in}}^{(2)}=-(-1)^{\gamma / \pi} \varphi_{\mathrm{in}}^{(1)}+\pi m_{1} / 2, m_{1}-\text { целое }\end{array}$ & $\begin{array}{l}\operatorname{Re}\left[\mathbf{f}^{(12)}(\theta,-\varphi) \exp \left(i m_{1} \pi / 2\right)\right]= \\
=\operatorname{Re}\left[\left(1-2 \mathbf{e}_{\varphi} \otimes \mathbf{e}_{\varphi}\right) \mathbf{f}^{(12)}(\theta, \varphi) \exp \left(i m_{1} \pi / 2\right)\right]\end{array}$ \\
\hline 9 & $\begin{array}{l}\chi_{1-5}^{(2)} \neq 0, \chi_{6,7}^{(2)}=0, \gamma=0, \theta_{\mathrm{in}}^{(1)}=\theta_{\mathrm{in}}^{(2)}, \\
\varphi_{\mathrm{in}}^{(2)}-\varphi_{\mathrm{in}}^{(1)}=\pi m_{1}, \sigma_{1}=\sigma_{2}=0, m_{1} \text { - целое }\end{array}$ & $\operatorname{Re}\left[\mathbf{f}^{(12)}(\theta, \varphi)\right]=0$ \\
\hline 10 & $\begin{array}{l}\chi_{1-5}^{(2)} \neq 0, \chi_{6,7}^{(2)}=0, \gamma=0, \pi \\
\theta_{\text {in }}^{(1)}=\gamma+(-1)^{\gamma / \pi} \theta_{\text {in }}^{(2)}, \sigma_{1}=\sigma_{2}=0\end{array}$ & $\operatorname{Re}\left[\mathbf{f}^{(12)}(\theta,-\varphi)\right]=\operatorname{Re}\left[\left(1-2 \mathbf{e}_{\varphi} \otimes \mathbf{e}_{\varphi}\right) \mathbf{f}^{(12)}(\theta, \varphi)\right]$ \\
\hline 11 & $\begin{array}{l}\chi_{1-5}^{(2)} \neq 0, \chi_{6,7}^{(2)}=0, \gamma=0, \pi, \theta_{\mathrm{in}}^{(2)} \pm \theta_{\mathrm{in}}^{(1)}=\frac{\pi \pm \pi}{2}, \\
\varphi_{\mathrm{in}}^{(2)}-(-1)^{\gamma / \pi} \varphi_{\mathrm{in}}^{(1)}=\pi m_{1}, \sigma_{1}=\sigma_{2}=0, m_{1} \text { - целое }\end{array}$ & $\operatorname{Re}\left[\mathbf{f}_{\text {lat }}^{(12)}(\theta,-\varphi)\right]=-\operatorname{Re}\left[\left(1-2 \mathbf{e}_{\varphi} \otimes \mathbf{e}_{\varphi}\right) \mathbf{f}_{\mathrm{lat}}^{(12)}(\theta, \varphi)\right]$ \\
\hline 12 & $\theta_{\mathrm{in}}^{(1)}=0, \pi, \theta_{\mathrm{in}}^{(2)}=0, \pi, m_{1}$ - целое & $\begin{array}{l}\mathbf{f}^{(12)}(-\theta, \varphi)=-\left(1-2 \mathbf{e}_{r} \otimes \mathbf{e}_{r}\right) \mathbf{f}^{(12)}\left(\theta, \varphi+\pi m_{1}\right) \\
S_{r}^{(12)}(-\theta, \varphi)=S_{r}^{(12)}\left(\theta, \varphi+\pi m_{1}\right)\end{array}$ \\
\hline 13 & $\theta_{\mathrm{in}}^{(1)}=0, \pi, \theta_{\mathrm{in}}^{(2)}=0, \pi, m_{1}$ - целое & $\begin{array}{l}\operatorname{Im}\left[\left(1-(1-i) \mathbf{e}_{\varphi} \otimes \mathbf{e}_{\varphi}\right) \mathbf{f}^{(12)}\left(\theta, \frac{\pi m_{1}+(-1)^{\frac{\theta_{\mathrm{in}}^{(1)}}{\pi}} \varphi_{\mathrm{in}}^{(1)}+(-1)^{\frac{\theta_{\mathrm{in}}^{(2)}}{\pi}} \varphi_{\mathrm{in}}^{(2)}}{2}-\varphi\right) \times\right. \\
\left.\times \exp \left(i m_{1} \pi / 2\right)\right]=\operatorname{Im}\left[\left(1-(1-i) \mathbf{e}_{\varphi} \otimes \mathbf{e}_{\varphi}\right)\right] \times \\
\left.\times \mathbf{f}^{(12)}\left(\theta, \frac{(-1)^{\frac{\theta_{\mathrm{in}}^{(1)}}{\pi}} \varphi_{\mathrm{in}}^{(1)}+(-1) \frac{\theta_{\mathrm{in}}^{(2)}}{\pi} \varphi_{\text {in }}^{(2)}}{2}+\varphi\right) \exp \left(i m_{1} \pi / 2\right)\right]\end{array}$ \\
\hline 14 & $\theta_{\mathrm{in}}^{(1)}=0, \pi, \theta_{\mathrm{in}}^{(2)}=0, \pi, \sigma_{1}=\sigma_{2}=0$ & $\operatorname{Re}\left[\mathbf{e}_{\varphi} \mathbf{f}^{(12)}(\theta, \varphi)\right]=0$ \\
\hline 15 & $\begin{array}{l}\theta_{\text {in }}^{(1)}=0, \pi, \theta_{\text {in }}^{(2)}=0, \pi,\left|\sigma_{1}\right|=\left|\sigma_{2}\right|=1 \\
\Delta \varphi-\text { действительное, } m_{1} \text { - целое, }\end{array}$ & $\begin{array}{l}\mathbf{f}^{(12)}(\theta, \varphi+\Delta \varphi)=\mathbf{f}^{(12)}\left(\theta, \pi m_{1}+\varphi\right) \times \\
\times \exp \left(i\left(\sigma_{1}(-1)^{\theta_{\mathrm{in}}^{(1)} / \pi}+\sigma_{2}(-1)^{\theta_{\mathrm{in}}^{(2)}} / \pi\right) \Delta \varphi\right), S_{r}^{(12)}(\theta, \varphi+\Delta \varphi)=S_{r}^{(12)}(\theta, \varphi)\end{array}$ \\
\hline 16 & $\begin{array}{l}\theta_{\mathrm{in}}^{(1)}=0, \pi, \theta_{\mathrm{in}}^{(2)}=0, \pi \\
\left|\sigma_{1}\right|=\left|\sigma_{2}\right|=1, m_{1} \text { - целое }\end{array}$ & $\begin{array}{l}i\left(1-(1-i) \mathbf{e}_{\theta} \otimes \mathbf{e}_{\theta}\right) \mathbf{f}^{(12)}(-\theta, \varphi)=\left[i\left(1-(1-i) \mathbf{e}_{\theta} \otimes \mathbf{e}_{\theta}\right) \mathbf{f}^{(12)}\left(\theta, \pi m_{1}-\varphi\right)\right. \\
\left.\times \exp \left(2 i\left(\sigma_{1} \varphi_{\mathrm{in}}^{(1)}+\sigma_{2} \varphi_{\mathrm{in}}^{(2)}\right)\right)\right]^{*}, S_{r}^{(12)}(-\theta, \varphi)=S_{r}^{(12)}\left(\theta, \pi m_{1}-\varphi\right)\end{array}$ \\
\hline
\end{tabular}


Таблица (продолжение).

\begin{tabular}{|c|c|c|}
\hline 1 & 2 & 3 \\
\hline 17 & $\begin{array}{l}\theta_{\mathrm{in}}^{(1)}=0, \pi, \theta_{\mathrm{in}}^{(2)}=0, \pi \\
\varphi_{\mathrm{in}}^{(2)}-(-1)^{\left(\theta_{\mathrm{in}}^{(1)}+\theta_{\mathrm{in}}^{(2)}\right) / \pi} \varphi_{\mathrm{in}}^{(1)}=m_{1} \pi / 2 \\
m=1,2, m_{1}, m_{2} \text { - целые }\end{array}$ & $\begin{array}{l}i\left(1-(1-i) \mathbf{e}_{\varphi} \otimes \mathbf{e}_{\varphi}\right) \mathbf{f}^{(12)}\left(\theta, \pi m_{2}-\varphi+(-1)^{\theta_{\mathrm{in}}^{(m)} / \pi} \varphi_{\mathrm{in}}^{(m)}\right) \exp \left(\frac{i m_{1} \pi}{2}\right)= \\
=\left[i\left(1-(1-i) \mathbf{e}_{\varphi} \otimes \mathbf{e}_{\varphi}\right) \mathbf{f}^{(12)}\left(\theta, \varphi+(-1)^{\theta_{\mathrm{in}}^{(m)} / \pi} \varphi_{\mathrm{in}}^{(m)}\right) \exp \left(\frac{i m_{1} \pi}{2}\right)\right], \\
S_{r}^{(12)}\left(\theta, \pi m_{2}-\varphi+(-1)^{\theta_{\mathrm{in}}^{(m)} / \pi} \varphi_{\mathrm{in}}^{(m)}\right)=S_{r}^{(12)}\left(\theta, \varphi+(-1)^{\theta_{\mathrm{in}}^{(m)} / \pi} \varphi_{\mathrm{in}}^{(m)}\right)\end{array}$ \\
\hline 18 & $\begin{array}{l}\chi_{2,5}^{(2)} \neq 0, \chi_{1,3,4,6,7}^{(2)}=0, \theta_{\mathrm{in}}^{(1)}=0, \pi, \theta_{\mathrm{in}}^{(2)}=0, \pi, \\
\Delta \varphi-\text { действительное }\end{array}$ & $\begin{array}{l}\mathbf{e}_{\varphi} \mathbf{f}^{(12)}(\theta, \varphi)=0, \mathbf{f}^{(12)}(\theta, \varphi+\Delta \varphi)=\mathbf{f}^{(12)}(\theta, \varphi) \\
S_{r}^{(12)}(\theta, \varphi+\Delta \varphi)=S_{r}^{(12)}(\theta, \varphi)\end{array}$ \\
\hline 19 & $\begin{array}{l}\chi_{1-5}^{2} \neq 0, \chi_{6,7}^{(2)}=0, \theta_{\mathrm{in}}^{(1)}=0, \pi, \theta_{\mathrm{in}}^{(2)}=0, \pi \\
\sigma_{1}= \pm \sigma_{2}, m_{1} \text { - целое }\end{array}$ & $\operatorname{Re}\left[\mathbf{f}_{\text {lat }}^{(12)}\left(\theta, \varphi+\pi m_{1}+\pi / 2\right)\right]=\operatorname{Re}\left[-\frac{\sigma_{1}}{\sigma_{2}}(-1)^{m_{1}} \mathbf{f}_{\text {lat }}^{(12)}(\theta, \varphi)\right]$ \\
\hline 20 & $\begin{array}{l}\chi_{1-4}^{2}=0, \chi_{5-7}^{(2)} \neq 0, \theta_{\mathrm{in}}^{(1)}=0, \pi, \theta_{\mathrm{in}}^{(2)}=0, \pi \\
\sigma_{1}= \pm \sigma_{2}, m_{1} \text { - целое }\end{array}$ & $\operatorname{Im}\left[\mathbf{f}^{(12)}\left(\theta, \varphi+\pi m_{1}+\pi / 2\right)\right]=\operatorname{Im}\left[-\frac{\sigma_{1}}{\sigma_{2}}(-1)^{\left(\theta_{m}^{(1)}+\theta_{m}^{(2)}\right) / \pi} \mathbf{f}^{(12)}(\theta, \varphi)\right]$ \\
\hline
\end{tabular}

В некоторых строках $(5,11,19)$ указана функция $\mathbf{f}_{\text {lat }}^{(12)}(\theta, \varphi)$ вместо $\mathbf{f}^{(12)}(\theta, \varphi)$. Это означает, что свойство выполняется лишь для функции, характеризующей генерацию от боковой поверхности, но не выполняется для функции, характеризующей генерацию от всей поверхности цилиндрической частицы.

Также стоит упомянуть о симметрии относительно перестановки падающих волн местами. Если произвести следующую замену:

$$
\begin{array}{r}
\mathbf{k}^{(1)} \leftrightarrow \mathbf{k}^{(2)}, \quad \mathbf{e}^{(1)} \leftrightarrow \mathbf{e}^{(2)}, \quad \chi_{3}^{(2)} \leftrightarrow \chi_{4}^{(2)}, \\
\chi_{5}^{(2)} \leftrightarrow-\chi_{5}^{(2)}, \quad \chi_{6}^{(2)} \leftrightarrow-\chi_{7}^{(2)}, \quad \chi_{7}^{(2)} \leftrightarrow-\chi_{6}^{(2)},
\end{array}
$$

то выражение для вектора $\mathbf{f}^{(12)}(\theta, \varphi)$ останется тем же.

\section{Графический анализ}

Проанализируем полученные зависимости графически. Для этого построим диаграммы направленности, характеризующие пространственное распределение генерируемого излучения. Лепестки диаграммы направленности показывают направления, в которых наблюдается наиболее интенсивная генерация.

Задача имеет более 20 параметров, поэтому для полного анализа задачи требуется проанализировать тысячи их возможных комбинаций. Это различные значения высот частицы $\left(k_{12} h \ll 1 / k_{12} h \sim 1 / k_{12} h \gg 1\right)$, радиусов основания $\left(k_{12} a \ll 1 / k_{12} a \sim 1 / k_{12} a \gg 1\right)$, коэффициентов анизотропии $\left(\chi_{1}^{(2)} \neq 0, \chi_{2-7}^{(2)}=0 / \chi_{2}^{(2)} \neq 0\right.$, $\chi_{1,3-7}^{(2)}=0 / \chi_{3}^{(2)} \neq 0, \quad \chi_{1-2,4-7}^{(2)} \quad$ и $\quad$ т.д.), угла раскрытия $\quad\left(\gamma=0 / \gamma=\frac{\pi}{2} / 0<\gamma<\frac{\pi}{2}\right), \quad$ соотношений между модулями волновых векторов падающих волн $\quad\left(\frac{k_{1}}{k_{12}} \ll 1 / \frac{k_{2}}{k_{12}} \ll 1 / k_{1} \approx k_{2}\right), \quad$ углов падения $\left(\theta_{\mathrm{in}}^{(1)}=0 / \theta_{\mathrm{in}}^{(1)}=\frac{\pi}{2} / 0<\theta_{\mathrm{in}}^{(1)}<\frac{\pi}{2}\right.$ и аналогично для $\left.\theta_{\mathrm{in}}^{(2)}\right)$, углов между большей полуосью эллипса поляризации и плоскостью падения $\left(\left|\varphi_{\text {in }}^{(1)}\right|=0 /\left|\varphi_{\text {in }}^{(1)}\right|=\frac{\pi}{2} / 0<\left|\varphi_{\text {in }}^{(1)}\right|<\right.$ $<\frac{\pi}{2}$ и аналогично для $\left.\varphi_{\text {in }}^{(2)}\right)$, степени эллиптичности падающего излучения $\quad\left(\left|\sigma_{1}\right|=0 /\left|\sigma_{1}\right|=1 / 0<\left|\sigma_{1}\right|<1\right.$ и аналогично для $\left.\left|\sigma_{2}\right|\right)$. Поэтому рассмотрены только отдельные комбинации параметров.

Далее приведены диаграммы направленности только для ключевых случаев. На их основе, а также на основе не приводимых здесь диаграмм направленности получены общие закономерности для генерации излучения суммарной частоты. Если в подписи рисунка не указано иное, то значения параметров по умолчанию следующие:

$$
\begin{gathered}
k_{1}=0.324 k_{12}, k_{2}=0.655 k_{12}, \sigma_{1}=0.5, \sigma_{2}=-0.3, \\
k_{12} a=1, k_{12} h=2 \pi, \theta_{\text {in }}^{(1)}=\theta_{\text {in }}^{(2)}=\pi / 2, \\
\varphi_{\text {in }}^{(1)}=0.1, \varphi_{\text {in }}^{(2)}=-0.6, \gamma=1.1 .
\end{gathered}
$$

Такие соотношения между модулями волновых векторов характерны для длин волн падающего излучения 850, $425 \mathrm{~nm}$ и длины волны суммарной частоты $283.3 \mathrm{~nm}$ в воде при $20^{\circ} \mathrm{C}$ (показатели преломления равны 1.3278 , 1.3414 и 1.3641 соответственно). При этом коэффициент $\xi$, согласно формуле (43), равен 1.022.

Исследуем вклад боковой и торцевых поверхностей в диаграмму направленности. Параметры генерации для рис. 3, $a-c$ отличаются только значениями коэффициентов анизотропии. На рис. 3, $a$ диаграмма направленности состоит из двух частей. Большой лепесток, направленный в левую часть рисунка, вызван генерацией от боковой поверхности цилиндрической частицы, а два малых лепестка, направленных в правую часть рисунка, вызваны генерацией от торцевой поверхности. На рис. 3, $b$ вклад боковой поверхности в диаграмму направленности настолько мал, что ее форма обусловлена только генерацией от торцевой поверхности, так как практически полностью совпадает с диаграммой направленности для генерации от торцов цилиндрической частицы (не приведена в работе). На рис. 3, с вклад торцевой поверхности оказался значительно меньше вклада боковой поверхности цилиндрической частицы, так как форма диаграммы направленности практически 


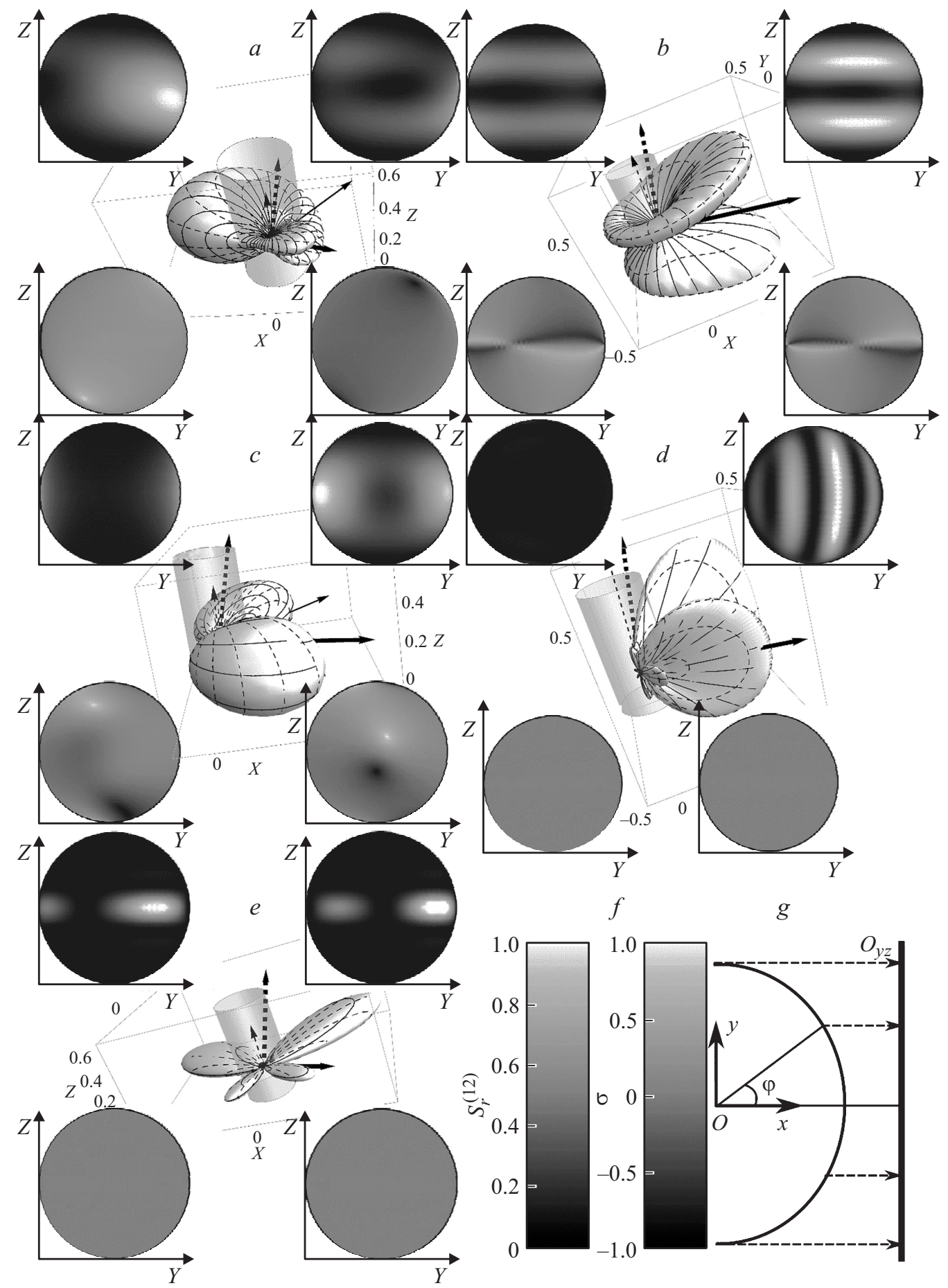

Рис. 3. Трехмерные нормированные диаграммы направленности для генерации суммарной частоты от цилиндрической частицы. Ориентация цилиндрической частицы показана на диаграмме направленности в виде прозрачного цилиндра. Тонкая (утолщенная) сплошная стрелка указывает на направление волнового вектора первой (второй) падающей волны. Тонкая (утолщенная) штриховая стрелка направлена вдоль большей полуоси эллипса поляризации первой (второй) падающей волны. Проекции больших (меньших) полуосей эллипса поляризации генерируемого излучения на поверхность диаграммы направленности обозначены сплошными (штриховыми) линиями на ее поверхности. Проекции диаграммы направленности на плоскость $O y z$ (левый для $\pi / 2<\varphi<3 \pi / 2$, правый для $-\pi / 2<\varphi<\pi / 2)$ показаны на двух графиках в верхней части диаграммы направленности. Белому цвету соответствует максимальная мощность излучения, а черному минимальная. Степень эллиптичности генерируемого излучения характеризуется графиками в нижней части диаграммы направленности с теми же правилами проецирования. Белому (черному) цвету соответствует право (лево) циркулярно поляризованое излучение. Легенда для графиков в углах диаграммы направленности представлена на рис. $3, f$, а схема проекции проиллюстрирована на рис. $3, g$. Параметры задачи, для которых построены диаграммы направленности: $(a) k_{12} a=1, k_{12} h=2 \pi, \chi_{3}^{(2)} \neq 0, \chi_{1-2,4-7}^{(2)}=0 ;(b) k_{12} a=1, k_{12} h=2 \pi, \chi_{1}^{(2)} \neq 0, \chi_{2-7}^{(2)}=0$; (c) $k_{12} a=1, k_{12} h=2 \pi, \chi_{5}^{(2)} \neq 0, \chi_{1-4,6-7}^{(2)}=0 ;(d) k_{12} a=5, k_{12} h=1, \chi_{1}^{(2)} \neq 0, \chi_{2-7}^{(2)}=0 ;(e) k_{12} a=1, k_{12} h=20, \chi_{1}^{(2)} \neq 0, \chi_{2-7}^{(2)}=0$. 


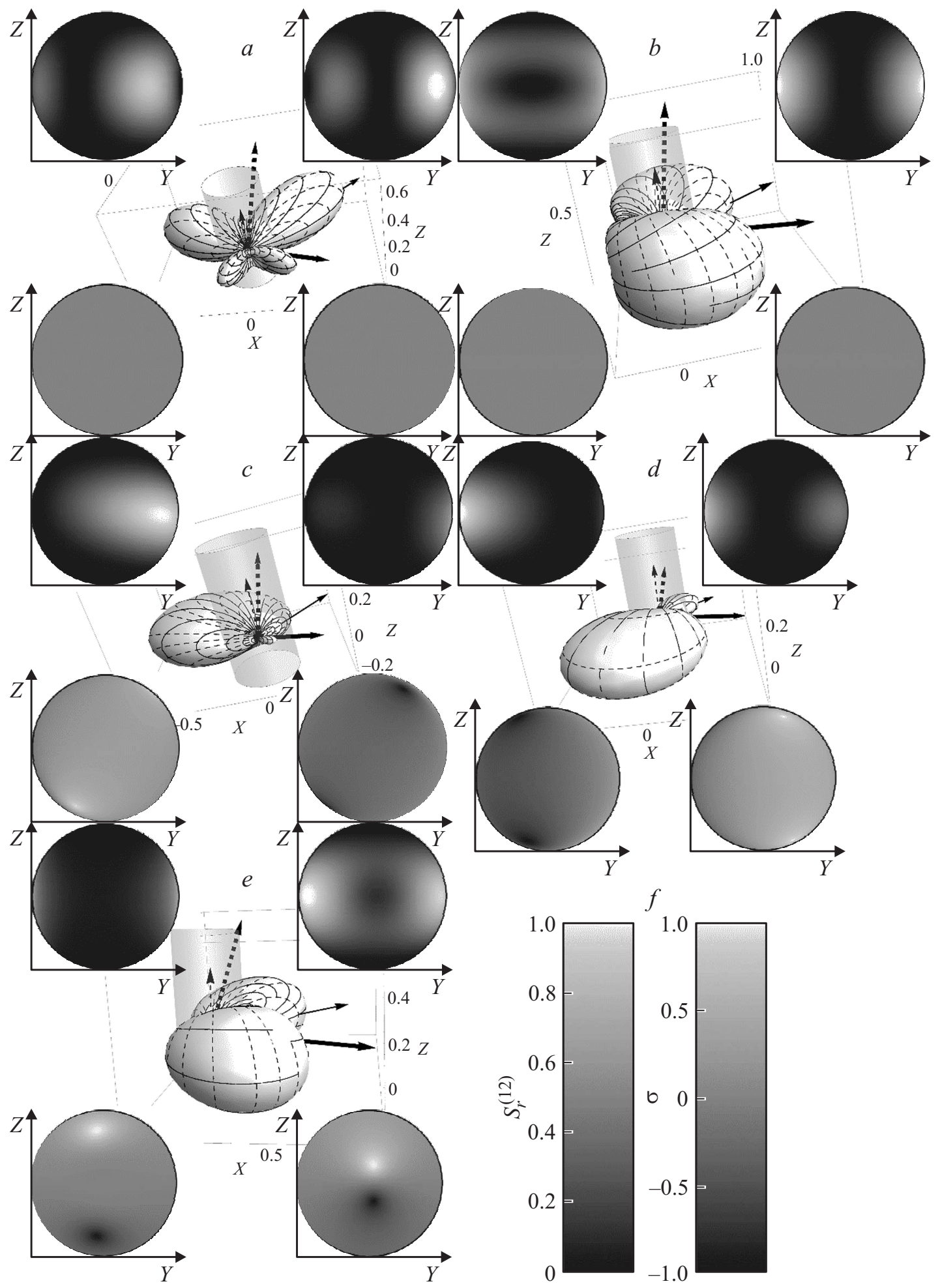

Рис. 4. Трехмерные нормированные диаграммы направленности для генерации суммарной частоты от боковой поверхности цилиндрической частицы. Обозначения аналогичны указанным в подписи к рис. 3. Параметры построения: $(a) \chi_{1}^{(2)} \neq 0, \chi_{2-7}^{(2)}=0$; (b) $\chi_{2}^{(2)} \neq 0, \chi_{1,3-7}^{(2)}=0 ;(c) \chi_{3}^{(2)} \neq 0, \chi_{1-2,4-7}^{(2)}=0 ;(d) \chi_{4}^{(2)} \neq 0, \chi_{1-3,5-7}^{(2)}=0 ;(e) \chi_{5}^{(2)} \neq 0, \chi_{1-4,6-7}^{(2)}=0$. Легенда представлена на рис. $4, f$.

полностью совпадает с формой диаграммы направленности для боковой поверхности частицы (также не приведена в работе). Таким образом, значения коэффициентов анизотропии $\chi_{1-7}^{(2)}$ нелинейного слоя сильно влияют на то, какая часть поверхности цилиндрической частицы определяет форму диаграммы направленности. Размеры 
частицы также влияют: увеличение ее высоты ведет к росту вклада боковой поверхности в форму диаграммы направленности.

Рассмотрим влияние размеров частицы на форму диаграммы направленности. На рис. 3,d изображена диаграмма направленности для генерации от боковой поверхности цилиндрической частицы с большим радиусом основания. При этом на диаграмме направленности выделилось несколько лепестков. Это объясняется тем, что в математическое выражение для поля суммарной частоты входят функции Бесселя, которые имеют на графике ряд максимумов и минимумов убывающей амплитуды. На диаграммах направленности для других значений коэффициентов анизотропии при увеличении радиуса основания частицы также выделяются главные и побочные лепестки.

На рис. 3,e изображена аналогичная диаграмма направленности, но для цилиндрической частицы большой высоты. При этом диаграмма направленности оказалась сплюснутой в направлении вдоль оси $O z$. Более того, на рисунке появились побочные лепестки, однако из-за своего малого размера они заметны только при его увеличении. Этот же эффект проявляется и для других значений коэффициентов анизотропии. Математически это объясняется предельной формой коэффициента (37).

Исследуем влияние коэффициентов анизотропии на форму диаграммы направленности (рис. 4). В большинстве случаев генерация от боковой поверхности цилиндрической частицы оказывает большее влияние на пространственное распределение излучения суммарной частоты, чем генерация от торцевой поверхности. Поэтому на рис. 4 изображены диаграммы направленности излучения, генерируемого от боковой поверхности цилиндра, для случаев, когда лишь один из коэффициентов анизотропии не равен нулю, кроме остальных (не равный нулю коэффициент указан в подписи рисунка). Можно видеть, что для каждого коэффициента анизотропии характерна своя форма диаграммы направленности. Часть из них имеет симметричную форму (рис. $4, a, b, e$ ), а часть асимметричную (рис. $4, c, d$ ). Экспериментально измерив пространственное распределение поля суммарной частоты, можно приблизительно оценить преобладающий вклад коэффициентов анизотропии в нелинейном слое.

На рис. 5 построены диаграммы направленности для различных значений углов падения и ориентации эллипсов поляризации падающего излучения. На рис. 5, $a$ волновые векторы падающих волн перпендикулярны оси цилиндра, а на рис. 5, $b$ располагаются под углом $\pi / 4$. Это отразилось и на форме диаграммы направленности. В первом случае оба лепестка направлены почти перпендикулярно оси цилиндра, а во втором лепестки отклонились вверх к направлению волновых векторов падающих волн. На рис. 5, c волновые векторы падающих волн направлены вдоль оси цилиндра. Это привело к тому, что лепестки также смещены в сторону волновых векторов, а сама диаграмма направленности приобрела зеркальную симметрию относительно двух плоскостей.
Таким образом, ориентация волновых векторов падающих волн оказывает непосредственное влияние на форму диаграммы направленности и смещение ее главных лепестков. При направлении волновых векторов вдоль оси цилиндрической частицы задача может приобретать осевую или зеркальную симметрию, что придает симметрию диаграммам направленности.

Рисунок 5, $d$ построен для тех же параметров задачи, что были использованы для рис. $5, b$, но эллипс поляризации первой волны повернут на угол $\pi / 4$, а второй на $-\pi / 3$. На диаграмме направленности рис. $5, d$ также присутствуют два лепестка, но они немного смещены относительно положения лепестков на рис. 5, $b$. Параметры задачи для рис. 5,e отличаются от параметров задачи для рис. $5, d$ только тем, что эллипс поляризации второй волны также повернут на угол $\pi / 4$. Оба лепестка на диаграмме направленности рис. 5,e стали немного шире по сравнению со случаем, изображенным на рис. $5, d$. Ориентация эллипса поляризации оказывает небольшое влияние на пространственное распределение излучения суммарной частоты. Но чем ближе степень эллиптичности падающей волны к \pm 1 , тем меньшее влияние оказывает угол поворота большей полуоси эллипса поляризации.

Следует также заметить, что при некоторых комбинациях параметров излучение суммарной частоты в определенных направлениях отсутствует (рис. $3, b, e, 4, a, c, d$, $5, a)$. Анализ таких направлений является достаточно сложной задачей, но они могут быть использованы для определения коэффициентов анизотропии $\chi_{1-7}^{(2)}$. Для определения этих коэффициентов могут быть также полезны сочетания параметров, при которых наблюдается линейно поляризованное излучение (рис. $3, d, e, 4, a, b)$. Например, если нелинейный слой не обладает киральными свойствами $\left(\chi_{5-7}^{(2)}=0\right)$, то при падении линейно поляризованных волн излучение суммарной частоты также имеет линейную поляризацию.

\section{Сравнение с работами других авторов}

В работе [10] рассмотрена та же задача, что и в нашей работе, но имеется несколько неточностей. В векторе рассеяния q в соответствии с выбранной временной зависимостью $\exp (-i \omega t)$ должен присутствовать множитель $(-1)$. Это влияет на распределение поля суммарной частоты в случае, когда среди коэффициентов $\chi_{1-4}^{(2)}$ и $\chi_{5-7}^{(2)}$ есть хотя бы по одному не равному нулю коэффициенту.

Также при вычислении интегралов для нахождения генерируемого поля от торцевых поверхностей цилиндрической частицы в [10] не учтено (как и в работе [14], посвященной генерации второй гармоники от цилиндрической частицы), что киральная часть тензора нелинейной восприимчивости $\chi_{5}^{(2)} n_{m} n_{i} \varepsilon_{m j k}+\chi_{6}^{(2)} n_{m} n_{k} \varepsilon_{i j m}+\chi_{7}^{(2)} n_{m} n_{j} \varepsilon_{i m k}$ не меняет своего знака при повороте вектора нормали на угол $\pi$. 


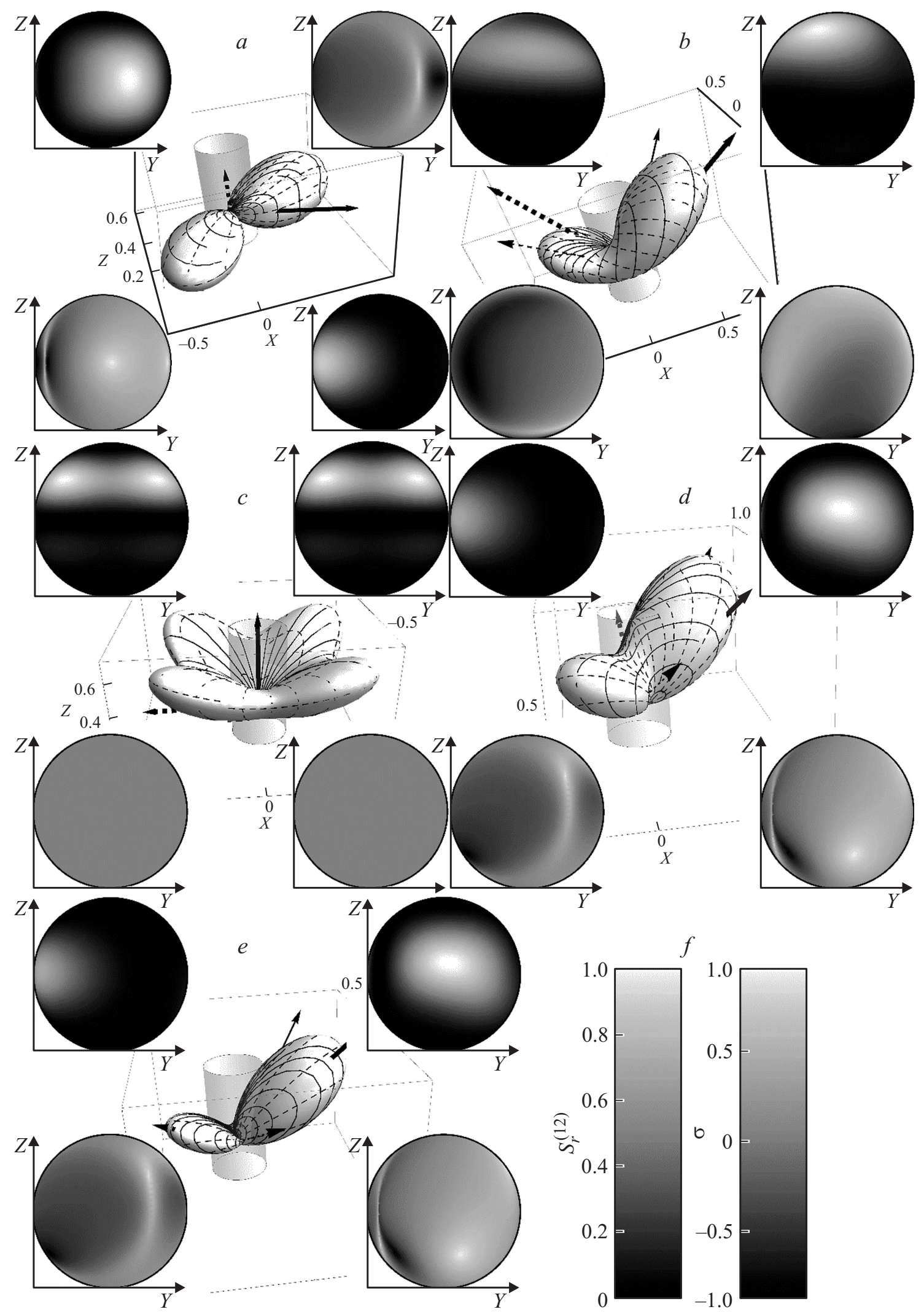

Рис. 5. Трехмерные нормированные диаграммы направленности для генерации суммарной частоты от боковой поверхности цилиндрической частицы. Обозначения аналогичны указанным в подписи к рис. 3. Параметры построения: $(a) \sigma_{1}=\sigma_{2}=0.5$, $k_{12} a=2, \varphi_{\mathrm{in}}^{(1)}=\varphi_{\mathrm{in}}^{(2)}=0, \chi_{6}^{(2)} \neq 0, \chi_{1-5,7}^{(2)}=0 ;(b) \sigma_{1}=\sigma_{2}=0.5, k_{12} a=2, \varphi_{\mathrm{in}}^{(1)}=\varphi_{\mathrm{in}}^{(2)}=0, \chi_{6}^{(2)} \neq 0, \chi_{1-5,7}^{(2)}=0, \theta_{\mathrm{in}}^{(1)}=\theta_{\mathrm{in}}^{(2)}=\pi / 4 ;$ (c) $\sigma_{1}=\sigma_{2}=0.5, k_{12} a=2, \varphi_{\mathrm{in}}^{(1)}=\varphi_{\mathrm{in}}^{(2)}=0, \chi_{6}^{(2)} \neq 0, \chi_{1-5,7}^{(2)}=0, \theta_{\mathrm{in}}^{(1)}=\theta_{\mathrm{in}}^{(2)}=0 ;(d) \sigma_{1}=\sigma_{2}=0.5, k_{12} a=2, \varphi_{\mathrm{in}}^{(1)}=\pi / 4, \varphi_{\mathrm{in}}^{(2)}=-\pi / 3$, $\chi_{6}^{(2)} \neq 0, \chi_{1-5,7}^{(2)}=0, \theta_{\mathrm{in}}^{(1)}=\theta_{\mathrm{in}}^{(2)}=\pi / 4 ;(e) \sigma_{1}=\sigma_{2}=0.5, k_{12} a=2, \varphi_{\mathrm{in}}^{(1)}=\varphi_{\mathrm{in}}^{(2)}=\pi / 4, \chi_{6}^{(2)} \neq 0, \chi_{1-5,7}^{(2)}=0, \theta_{\mathrm{in}}^{(1)}=\theta_{\mathrm{in}}^{(2)}=\pi / 4$. 
Это привело к появлению в формуле для поля от торцов цилиндрической частицы (26) мнимого коэффициента $i \sin \left(q_{\|} h / 2\right)$ вместо действительного $\cos \left(q_{\|} h / 2\right)$. Тогда при использовании функции $\cos \left(q_{\|} h / 2\right)$ выражение для поля суммарной частоты, обусловленного киральными компонентами тензора $\chi_{i j k}^{(2)}$, будет отличаться по фазе от выражения для поля, обусловленного некиральными компонентами, на множитель $i$ так же, как и при генерации от боковой поверхности. При малых радиусах основания цилиндра $q_{\perp} a \ll 1$ вклад киральных компонент в генерацию от боковой поверхности цилиндра является преобладающим. В предельном случае $q_{\|} h \ll 1$ киральные компоненты также вносят решающий вклад в распределение поля суммарной частоты от торцов, за что отвечает функция $\cos \left(q_{\|} h / 2\right)$ в формуле (26). Полученная в работе [10] функция $i \sin \left(q_{\|} h / 2\right)$ приводит к отсутствию этого свойства. Тогда после такого уточнения (замены $i \sin \left(q_{\|} h / 2\right)$ на $\cos \left(q_{\|} h / 2\right)$ ) коэффициент $B_{z^{\prime} z^{\prime}}$ в табл. 4 работы [10] примет значение

$$
B_{z^{\prime} z^{\prime}}=2 \pi D^{2}\left(J_{0}\left(q_{x^{\prime}} D\right)+J_{2}\left(q_{x^{\prime}} D\right)\right) \cos \left(q_{z^{\prime}} L / 2\right) .
$$

С учетом этих исправлений связь между обозначениями авторов в [10] и нашими запишется в виде

$$
\begin{aligned}
& A_{i j k}(\mathbf{q})=2 \pi i a h \frac{\sin \left(q_{\|} h / 2\right)}{q_{\|} h / 2}\left[-J_{3}\left(q_{\perp} a\right) v_{i} v_{j} v_{k}\right. \\
&+\frac{1}{4}\left(J_{1}\left(q_{\perp} a\right)+J_{3}\left(q_{\perp} a\right)\right)\left(v_{i}\left(\delta_{j k}-\delta_{j z} \delta_{k z}\right)\right. \\
&\left.\left.+v_{j}\left(\delta_{k i}-\delta_{i z} \delta_{k z}\right)+v_{k}\left(\delta_{i j}-\delta_{i z} \delta_{j z}\right)\right)\right] \\
&+2 \pi i a^{2}\left(J_{0}\left(q_{\perp} a\right)+J_{2}\left(q_{\perp} a\right)\right) \sin \left(q_{\|} h / 2\right) e_{z, i} e_{z, j} e_{z, k}, \\
& B_{i j}(\mathbf{q})=2 \pi a h \frac{\sin \left(q_{\|} h / 2\right)}{q_{\|} h / 2} \times\left(-v_{i} v_{j} J_{2}\left(q_{\perp} a\right)+\left(\delta_{i j}-\delta_{i z} \delta_{j z}\right) \frac{1}{2}\left(J_{0}\left(q_{\perp} a\right)+J_{2}\left(q_{\perp} a\right)\right)\right) \\
&+2 \pi a^{2}\left(J_{0}\left(q_{\perp} a\right)+J_{2}\left(q_{\perp} a\right)\right) \cos \left(q_{\|} h / 2\right) e_{z, i} e_{z, j}, \\
& C_{k}(\mathbf{q})=2 \pi i a h \frac{\sin \left(q_{\|} h / 2\right)}{q_{\|} h / 2} J_{1}\left(q_{\perp} a\right) v_{k} \\
& \quad+2 \pi i a^{2}\left(J_{0}\left(q_{\perp} a\right)+J_{2}\left(q_{\perp} a\right)\right) \sin \left(q_{\|} h / 2\right) e_{z, k} .
\end{aligned}
$$

Кроме того, для полученного нами решения выполняются общие свойства, описанные в [10]:

$$
\begin{gathered}
A_{j k k}=C_{j}, \\
C_{k}=i q_{k} \int_{V} \exp \left(i \mathbf{q} \mathbf{x}^{\prime}\right) d^{3} \mathbf{x}^{\prime}=i q_{k} 4 \pi a \frac{\sin \left(q_{\|} h / 2\right)}{q_{\|}} \frac{J_{1}\left(q_{\perp} a\right)}{q_{\perp}},
\end{gathered}
$$

$$
\begin{aligned}
B_{k k} & =\int_{S} \exp \left(i \mathbf{q} \mathbf{x}^{\prime}\right) d^{2} \mathbf{x}^{\prime} \\
& =4 \pi a\left(\frac{J_{1}\left(q_{\perp} a\right)}{q_{\perp}} \cos \left(q_{\|} h / 2\right)+\frac{J_{0}\left(q_{\perp} a\right)}{q_{\|}} \sin \left(q_{\|} h / 2\right)\right),
\end{aligned}
$$

где под повторяющимися индексами в $A, B, C$ подразумевается свертка, а $\int_{V} \exp \left(i \mathbf{q} \mathbf{x}^{\prime}\right) d^{3} \mathbf{x}^{\prime}$ и $\int_{S} \exp \left(i \mathbf{q} \mathbf{x}^{\prime}\right) d^{2} \mathbf{x}^{\prime}-$ линейные объемный и поверхностный формфакторы соответственно [10]. Нетрудно проверить, что эти свойства будут выполняться и для случая неверного учета киральных коэффициентов $\chi_{5-7}^{(2)}$ в вычисляемых интегралах.

\section{Заключение}

Обобщенное приближение Релея-Ганса-Дебая существенно упрощает задачу о генерации гармоники суммарной частоты. Полученные благодаря ему формулы описывают и пространственное распределение, и поляризацию генерируемого излучения. Представление решения как в тензорной, так и векторной формах значительно упростило анализ предельных случаев, свойств симметрии полученного решения и трехмерных диаграмм направленности генерируемого излучения суммарной частоты, так как отпадает необходимость иметь дело с множеством значений отдельных компонент тензоров.

Подробный анализ показал, что функции, характеризующие пространственное распределение поля суммарной частоты, имеют ряд математических свойств, перечисленных в таблице, которые приводят к различным симметриям диаграмм направленности при некоторых комбинациях параметров задачи. На поляризацию генерируемого излучения оказывает сильное влияние поляризация падающего излучения. В частности, для некирального слоя линейно поляризованные падающие волны приводят к генерации линейно поляризованного излучения. Рассмотрение эллиптичности в качестве одного из дополнительных варьируемых параметров позволит извлечь больше информации при спектроскопии, основанной на генерации суммарной частоты.

Преобладание вклада киральных компонент тензора нелинейной диэлектрической восприимчивости в генерацию при малых размерах частицы от торцов позволяет оценить значения киральных компонент для небольших цилиндрических частиц с произвольным соотношением радиуса основания и высоты частицы. Ранее этот эффект предсказывался только для боковой поверхности цилиндрических частиц.

Полученное в работе аналитическое решение может быть использовано также и для описания генерации второй гармоники от поверхности цилиндрических частиц. В этом случае количество варьируемых параметров сокращается, что позволит провести значительно более подробный анализ. 


\section{Приложение A}

Введем систему отсчета, в которой ось $O x^{\prime}$ направлена вдоль $\mathbf{q}_{\perp}$. В таком случае старые базисные векторы $\left(\mathbf{e}_{x}, \mathbf{e}_{y}, \mathbf{e}_{z}\right)$ выразятся через новые $\left(\mathbf{e}_{x}^{\prime}, \mathbf{e}_{y}^{\prime}, \mathbf{e}_{z}^{\prime}\right)$ следующим образом:

$$
\left[\begin{array}{l}
\mathbf{e}_{x} \\
\mathbf{e}_{y} \\
\mathbf{e}_{z}
\end{array}\right]=\left[\begin{array}{ccc}
\cos \varphi_{q} & -\sin \varphi_{q} & 0 \\
\sin \varphi_{q} & \cos \varphi_{q} & 0 \\
0 & 0 & 1
\end{array}\right]\left[\begin{array}{l}
\mathbf{e}_{x}^{\prime} \\
\mathbf{e}_{y}^{\prime} \\
\mathbf{e}_{z}^{\prime}
\end{array}\right]
$$

где $\varphi_{q}-$ это угол между $\mathbf{q}_{\perp}$ и осью $O x^{\prime}$ в старой системе отсчета. Тогда в новой системе координат вектор $\mathbf{n}$ запишется в виде

$$
\mathbf{n}=\cos \varphi^{\prime} \mathbf{e}_{x}^{\prime}+\sin \varphi^{\prime} \mathbf{e}_{y}^{\prime} .
$$

Проекции этого вектора на старые базисные векторы следующие:

$$
\begin{gathered}
\mathbf{n e}_{x}=\cos \varphi_{q} \cos \varphi^{\prime}-\sin \varphi_{q} \sin \varphi^{\prime}=\cos \left(\varphi^{\prime}+\varphi_{q}\right), \\
\mathbf{n e}_{y}=\sin \varphi_{q} \cos \varphi^{\prime}+\cos \varphi_{q} \sin \varphi^{\prime}=\sin \left(\varphi^{\prime}+\varphi_{q}\right), \\
\mathbf{n e}_{z}=0 .
\end{gathered}
$$

При дальнейших вычислениях понадобятся вспомогательные интегралы $(m \geq 0$ - целое)

$$
\begin{gathered}
\int_{0}^{2 \pi} \exp \left(i z \cos \varphi^{\prime}\right) \cos ^{m} \varphi^{\prime} d \varphi^{\prime}=\frac{2 \pi}{i^{m}} J_{0}^{(m)}(z), \\
\int_{0}^{2 \pi} \exp \left(i z \cos \varphi^{\prime}\right) \cos ^{m} \varphi^{\prime} \sin \varphi^{\prime} d \varphi^{\prime}=0 .
\end{gathered}
$$

Здесь верхний индекс $(m)$ означает производную порядка $m$ по аргументу. Также необходимо будет воспользоваться свойствами цилиндрических функций Бесселя:

$$
2 J_{m}^{(1)}(z)=J_{m-1}(z)-J_{m+1}(z) .
$$

Пользуясь (53), получим для интеграла в (15) при $k=x$

$$
\begin{aligned}
\int_{0}^{2 \pi} & \exp (i a \mathbf{q} \perp \mathbf{n}) n_{x} d \varphi^{\prime}=\int_{0}^{2 \pi} \exp \left(i a \mathbf{q}_{\perp} \mathbf{n}\right)\left(\mathbf{n e}_{x}\right) d \varphi^{\prime} \\
\quad & \int_{0}^{2 \pi} \exp \left(i q_{\perp} a \cos \varphi^{\prime}\right)\left(\cos \varphi_{q} \cos \varphi^{\prime}-\sin \varphi_{q} \sin \varphi^{\prime}\right) d \varphi^{\prime} \\
= & \cos \varphi_{q}\left(-2 \pi i J_{0}^{(1)}\left(q_{\perp} a\right)\right)=-2 \pi i v_{x} J_{0}^{(1)}\left(q_{\perp} a\right) .
\end{aligned}
$$

Аналогичные формулы получаем при вычислении интегралов с $k=y$ и $k=z$. Затем, воспользовавшись (55), получаем формулу (15).
Для вычисления интеграла (16) также понадобится перейти в новую систему отсчета. Рассмотрим этот интеграл поочередно для различных значений индексов. Пусть $i=j=x$, тогда

$$
\begin{aligned}
& \int_{0}^{2 \pi} \exp \left(i a \mathbf{q}_{\perp} \mathbf{n}\right) n_{x}^{2} d \varphi^{\prime}=\int_{0}^{2 \pi} \exp \left(i a \mathbf{q}_{\perp} \mathbf{n}\right)\left(\mathbf{n} \mathbf{e}_{x}\right)^{2} d \varphi^{\prime} \\
& =\int_{0}^{2 \pi} \exp \left(i q_{\perp} a \cos \varphi^{\prime}\right)\left(\cos \varphi_{q} \cos \varphi^{\prime}-\sin \varphi_{q} \sin \varphi^{\prime}\right)^{2} d \varphi^{\prime} \\
& =\cos ^{2} \varphi_{q} \int_{0}^{2 \pi} \exp \left(i q_{\perp} a \cos \varphi^{\prime}\right) \cos ^{2} \varphi^{\prime} d \varphi^{\prime} \\
& \quad+\sin ^{2} \varphi_{q} \int_{0}^{2 \pi} \exp \left(i q_{\perp} a \cos \varphi^{\prime}\right)\left(1-\cos ^{2} \varphi^{\prime}\right) d \varphi^{\prime} \\
& =2 \pi\left[-\cos ^{2} \varphi_{q} J_{0}^{(2)}\left(q_{\perp} a\right)+\sin ^{2} \varphi_{q}\left(J_{0}\left(q_{\perp} a\right)+J_{0}^{(2)}\left(q_{\perp} a\right)\right)\right] \\
& =2 \pi\left[-v_{x}^{2} J_{0}^{(2)}\left(q_{\perp} a\right)+\left(1-v_{x}^{2}\right)\left(J_{0}\left(q_{\perp} a\right)+J_{0}^{(2)}\left(q_{\perp} a\right)\right)\right] .
\end{aligned}
$$

Аналогично для $i=x, j=y$

$$
\int_{0}^{2 \pi} \exp \left(i a \mathbf{q}_{\perp} \mathbf{n}\right) n_{x} n_{y} d \varphi^{\prime}=\int_{0}^{2 \pi} \exp \left(i a \mathbf{q}_{\perp} \mathbf{n}\right)\left(\mathbf{n e}_{x}\right)\left(\mathbf{n e}_{y}\right) d \varphi^{\prime}
$$$$
=\int_{0}^{2 \pi} \exp \left(i q_{\perp} a \cos \varphi^{\prime}\right)\left(\cos \varphi_{q} \cos \varphi^{\prime}-\sin \varphi_{q} \sin \varphi^{\prime}\right)
$$$$
\times\left(\sin \varphi_{q} \cos \varphi^{\prime}+\cos \varphi_{q} \sin \varphi^{\prime}\right) d \varphi^{\prime}=\cos \varphi_{q} \sin \varphi_{q}
$$$$
\times \int_{0}^{2 \pi} \exp \left(i q a \cos \varphi^{\prime}\right) \cos ^{2} \varphi^{\prime} d \varphi^{\prime}-\cos \varphi_{q} \sin \varphi_{q}
$$

$$
\begin{aligned}
& \times \int_{0}^{2 \pi} \exp \left(i q a \cos \varphi^{\prime}\right)\left(1-\cos ^{2} \varphi^{\prime}\right) d \varphi^{\prime}=-2 \pi \cos \varphi_{q} \sin \varphi_{q} \\
& -2 \pi \cos \varphi_{q} \sin \varphi_{q}\left(J_{0}\left(q_{\perp} a\right)+J_{0}^{(2)}\left(q_{\perp} a\right)\right) \\
& =2 \pi\left[-v_{x} v_{y} J_{0}^{(2)}\left(q_{\perp} a\right)-v_{x} v_{y}\left(J_{0}\left(q_{\perp} a\right)+J_{0}^{(2)}\left(q_{\perp} a\right)\right)\right] .
\end{aligned}
$$

Далее, вычисляя этот интеграл для других значений индексов и пользуясь (55), обобщаем результаты в виде формулы (16). 
Интеграл (17) также можно вычислить в новой системе координат для всех значений индексов. Рассмотрим некоторые из них. Пусть $i=j=k=x$, тогда

$$
\begin{aligned}
& \int_{0}^{2 \pi} \exp \left(i a \mathbf{q}_{\perp} \mathbf{n}\right) n_{x}^{3} d \varphi^{\prime}=\int_{0}^{2 \pi} \exp \left(i a \mathbf{q}_{\perp} \mathbf{n}\right)\left(\mathbf{n} \mathbf{e}_{x}\right)^{3} d \varphi^{\prime} \\
& =\int_{0}^{2 \pi} \exp \left(i q_{\perp} a \cos \varphi^{\prime}\right)\left(\cos \varphi_{q} \cos \varphi^{\prime}-\sin \varphi_{q} \sin \varphi^{\prime}\right)^{3} d \varphi^{\prime} \\
& =\cos ^{3} \varphi_{q} \int_{0}^{2 \pi} \exp \left(i q_{\perp} a \cos \varphi^{\prime}\right) \cos ^{3} \varphi^{\prime} d \varphi^{\prime} \\
& +3 \cos \varphi_{q} \sin ^{2} \varphi_{q} \int_{0}^{2 \pi} \exp \left(i q_{\perp} a \cos \varphi^{\prime}\right) \cos \varphi^{\prime}\left(1-\cos ^{2} \varphi^{\prime}\right) d \varphi^{\prime} \\
& =\cos { }^{3} \varphi_{q} 2 \pi i J_{0}^{(3)}\left(q_{\perp} a\right)+3 \cos \varphi_{q} \sin ^{2} \varphi_{q}(-2 \pi i) J_{0}^{(1)}\left(q_{\perp} a\right) \\
& -3 \cos \varphi_{q} \sin ^{2} \varphi_{q} 2 \pi i J_{0}^{(3)}\left(q_{\perp} a\right)=2 \pi i\left[v _ { x } ^ { 3 } \left(3 J_{0}^{(1)}\left(q_{\perp} a\right)\right.\right. \\
& \left.\left.+4 J_{0}^{(3)}\left(q_{\perp} a\right)\right)-3 v_{x}\left(J_{0}^{(1)}\left(q_{\perp} a\right)+J_{0}^{(3)}\left(q_{\perp} a\right)\right)\right] .
\end{aligned}
$$

Аналогично для $i=x, j=x, k=y$

$$
\begin{aligned}
& \int_{0}^{2 \pi} \exp \left(i a \mathbf{q}_{\perp} \mathbf{n}\right) n_{x}^{2} n_{y} d \varphi^{\prime}=\int_{0}^{2 \pi} \exp \left(i a \mathbf{q}_{\perp} \mathbf{n}\right)\left(\mathbf{n e}_{x}\right)^{2}\left(\mathbf{n e}_{y}\right) d \varphi^{\prime} \\
& =\int_{0}^{2 \pi} \exp \left(i q_{\perp} a \cos \varphi^{\prime}\right)\left(\cos \varphi_{q} \cos \varphi^{\prime}\right. \\
& \left.-\sin \varphi_{q} \sin \varphi^{\prime}\right)^{2}\left(\sin \varphi_{q} \cos \varphi^{\prime}+\cos \varphi_{q} \sin \varphi^{\prime}\right) d \varphi^{\prime} \\
& =3 \cos ^{2} \varphi_{q} \sin \varphi_{q} \int_{0}^{2 \pi} \exp \left(i q_{\perp} a \cos \varphi^{\prime}\right) \cos ^{3} \varphi^{\prime} d \varphi^{\prime} \\
& -2 \cos ^{2} \varphi_{q} \sin \varphi_{q} \int_{0}^{2 \pi} \exp \left(i q_{\perp} a \cos \varphi^{\prime}\right) \cos \varphi^{\prime} d \varphi^{\prime} \\
& +\sin ^{3} \varphi_{q} \int_{0}^{2 \pi} \exp \left(i q_{\perp} a \cos \varphi^{\prime}\right) \cos \varphi^{\prime} d \varphi^{\prime}-\sin ^{3} \varphi_{q} \\
& \times \int_{0}^{2 \pi} \exp \left(i q_{\perp} a \cos \varphi^{\prime}\right) \cos ^{3} \varphi^{\prime} d \varphi^{\prime}=3 \cos ^{2} \varphi_{q} \sin \varphi_{q} \\
& \times 2 \pi i J_{0}^{(3)}\left(q_{\perp} a\right)+2 \cos ^{2} \varphi_{q} \sin \varphi_{q} 2 \pi i J_{0}^{(1)}\left(q_{\perp} a\right) \\
& -\sin ^{3} \varphi_{q} 2 \pi i J_{0}^{(1)}\left(q_{\perp} a\right)-\sin ^{3} \varphi_{q} 2 \pi i J_{0}^{(3)}\left(q_{\perp} a\right) \\
& =2 \pi i\left[v_{x}^{2} v_{y}\left(3 J_{0}^{(1)}\left(q_{\perp} a\right)+4 J_{0}^{(3)}\left(q_{\perp} a\right)\right)\right. \\
& \left.-v_{y}\left(J_{0}^{(1)}\left(q_{\perp} a\right)+J_{0}^{(3)}\left(q_{\perp} a\right)\right)\right] \text {. }
\end{aligned}
$$

Вычисляя интегралы для других индексов, обобщая полученные результаты и пользуясь (55), получаем выражение (17).

\section{Список литературы}

[1] Hartings J.M., Poon A., Pu X., Chang R.K., Leslie T.M. // Chem. Phys. Lett. 1997. V. 281. P. 389-393. doi 10.1016/S0009-2614(97)01315-8

[2] Roke S., Gonella G. // Ann. Rev. Phys. Chem. 2012. V. 63. P. 353-378. doi 10.1146/annurev-physchem-032511-143748

[3] Wang H., Troxler T., Yeh A.G., Dai H.L. // Langmuir. 2000. V. 16. P. 2475-2481. doi 10.1021/1a9909087

[4] Eckenrode M., Dai H.L. // Langmuir. 2004. V. 20. P. 9202 9209. doi 10.1021/la048863j

[5] Gonella G., Dai H.L. // Langmuir. 2014. V. 30. P. 2588-2599. doi10.1021/la403570f

[6] Liu J., Subir M., Nguyen K., Eisenthal K.B. // J. Phys. Chem. B. 2008. V. 112. N 48. P. 15263-15266. doi $10.1021 / \mathrm{jp} 806690 \mathrm{z}$

[7] Dadap J.I., Eisenthal K.B. // J. Phys. Chem. B. 2014. Vol. 118. N 49. doi 10.1021/jp507834s

[8] Viarbitskaya S., Kapshai V., P.v.d. Meulen, Hansson T. // Phys. Rev. A. 2011. V. 81. P. 053850. doi 10.1103/PhysRevA.81.053850

[9] de Beer A.G.F., Roke S. // Phys. Rev. B. 2007. V. 75. N 24. P. 245438. doi 10.1103/PhysRevB.75.245438

[10] de Beer A.G.F., Roke S., Dadap J.I. // J. Opt. Soc. Am. B. 2011. V. 28. N 6. P. 1374-1384. doi 10.1364/JOSAB.28.001374

[11] de Beer A.G.F., Roke S. // Phys. Rev. B. 2009. V. 79. N 15. P. 155420. doi 10.1103/PhysRevB.79.155420

[12] Wunderlich S., Schü rer B., Sauerbeck C., Peukert W., Peschel U. // Phys. Rev. B. 2011. V. 84. P. 235403. doi 10.1103/PhysRevB.84.235403

[13] Roke S., Bonn M., Petukhov A.V. // Phys. Rev. B. 2004. V. 70. N 11. P. 115106. doi 10.1103/PhysRevB.70.115106

[14] Dadap J.I. // Phys. Rev. B. 2008. V. 78. N 20. P. 205322. doi 10.1103/PhysRevB.78.205322 